\title{
Numerical simulation of polygonal particles moving in incompressible viscous fluids
}

\author{
Xiaowu $\mathrm{Fu}^{\mathrm{a}}$, Zhaohui Yao ${ }^{\mathrm{a}, *}$, Xiwen Zhang ${ }^{\mathrm{a}}$ \\ ${ }^{a}$ Department of Engineering Mechanics, Tsinghua University, Beijing 100084, China \\ *Corresponding author. E-mail: yaozh@tsinghua.edu.cn
}

\begin{abstract}
A two-dimensional coupled lattice Boltzmann immersed boundary discrete element method is introduced for the simulation of polygonal particles moving in incompressible viscous fluids. A collision model of polygonal particles is used in the discrete element method. Instead of a collision model of circular particles, the collision model used in our method can deal with particles of more complex shape and efficiently simulate the effects of shape on particle-particle and particle-wall interactions. For two particles falling under gravity, because of the edges and corners, different collision patterns for circular and polygonal particles are found in our simulations. The complex vortexes generated near the corners of polygonal particles affect the flow field and lead to a difference in particle motions between circular and polygonal particles. For multiple particles falling under gravity, the polygonal particles easily become stuck owing to their corners and edges, while circular particles slip along contact areas. The present method provides an efficient approach for understanding the effects of particle shape on the dynamics of non-circular particles in fluids.
\end{abstract}

Keywords: Polygonal particle; Particle-fluid interaction; Lattice Boltzmann method; Immersed boundary method; Discrete element method

\section{Introduction}

Particles moving in fluids are commonly found in nature, such as when plant seeds move in the wind and pebbles move in a river. Many industrial processes, including chemical, pharmaceutical, and mineral processes, also involve the transport of solid particles by fluids. Understanding the mechanism and behavior of particles in fluids is important in terms of being able to control or optimize particulate flows. Particulate flow commonly encountered in nature or industry is often complicated since non-spherical particles often 
exist. Recently, non-spherical particles have received attention because of their application to granular systems of academic and industrial interest.

When particles move in fluids, the particle shapes may affect the flow field in addition to there being particle-particle contact. Collisions between particles are always possible during the movement of multiple particles in fluids. Actual particles may have complex geometrical shapes and the collision and migration of particles may be highly complicated. The behaviors of non-circular or non-spherical particles moving in a flow may be greatly different from those of circular or spherical particles. With the development of computing technology, numerical simulations have come to play an important role in the investigation of particles moving in fluids. Numerical simulation can provide details for exploring the fundamental mechanisms of particles in fluids when experimental measurements are difficult to make (Xiong et al., 2010). By solving translational and rotational equations for particle motion and Navier-Stokes (N-S) equations for the fluid phase, Challabotla, Zhao, and Andersson (2015) performed numerical simulations of inertial disks in a turbulent channel flow. They found that disks can be made to preferentially align either parallel or perpendicular to the wall depending on the particle density. Voth (2015) found that particle shape also affects alignment, particularly for particles of lower density, and the alignment of disks is different from the alignment of fibers.

In recent years, as an alternative computational method of solving $\mathrm{N}-\mathrm{S}$ equations, the lattice Boltzmann method (LBM) has been widely used in the simulation of a flow field owing to its simplicity and intrinsic parallel nature. In addition, the LBM has become popular in the simulation of particulate flows owing to its particle-based numerical scheme, which calculates the dynamics of fictitious particles (Yu \& Fan, 2010).

Combining Newtonian dynamics of the solid particles with a discretized Boltzmann equation for the fluid phase, Ladd (1994a, 1994b) presented a general and effective technique of simulating solid-fluid suspensions. The application of the LBM to simulate particle-fluid suspensions was reviewed by Ladd and Verberg (2001). However, the non-slip condition on the particle-fluid interface is treated by the bounce-back rule in the LBM, which needs many particle boundary nodes to accurately represent the particle physical boundaries. In addition, the forces and velocities of the particles may fluctuate when the bounce-back 
boundary conditions are applied. Noble and Torczynski (1998) proposed a modified LBM that avoids step-like solutions produced using the bounce-back condition. Using the method proposed by Noble and Torczynski (1998), Xiong et al. (2012a, 2012b) conducted large-scale direct numerical simulations of gas-solid flows.

The immersed boundary method (IBM), which was first introduced by Peskin (1977) to study blood flow in the heart, has received great attention in recent years. Peskin (2002) provided the mathematical structure of the IBM. The IBM is simple and flexible for the simulation of flows with complex and moving boundaries. In the IBM, the solid is considered as an immersed boundary, which affects the flow through the boundary. The solid migrates with the flow owing to the non-slip condition on the boundary. Uhlmann (2005) presented a direct-forcing scheme based on the $\mathrm{N}-\mathrm{S}$ equations for computing incompressible viscous flow around suspended rigid particles using a fixed and uniform computational grid. This method allows for a smooth transfer between Eulerian and Lagrangian representations while avoiding strong restrictions of the time step. Di and Ge (2015) proposed an improved direct-forcing immersed boundary method for the simulation of fluid-solid interactions.

Combining most features of the LBM and IBM, a two-dimensional immersed boundary-lattice Boltzmann method (IB-LBM) was presented to solve fluid-particle interaction problems by Feng and Michaelides (2004). The IB-LBM uses two unrelated computational meshes, a Eulerian mesh for the flow domain and a Lagrangian mesh for the solid boundaries in the flow. The restoration force due to deformation is computed by the penalty method which uses a user-defined spring parameter (Feng \& Michaelides, 2004). Niu, Shu, Chew, and Peng (2006) presented a two-dimensional momentum exchange-based IB-LBM for simulating particles in incompressible viscous flows. In their method, a forcing term is introduced into the lattice Boltzmann equation to enforce the non-slip boundary. The forcing term is simply calculated from the momentum exchange of the boundary particle density distribution functions, which are interpolated using Lagrangian polynomials from the underlying Eulerian mesh. Employing the IB-LBM, Shu, Liu, and Chew (2007) proposed a velocity correction approach for the simulation of two-dimensional flow over a circular cylinder. This approach directly corrects the velocity to enforce the physical boundary condition. It is simple in concept and easy to implement. Employing the lattice Boltzmann equation with an external forcing term 
proposed by Guo, Zheng, and Shi (2002), Wu and Shu (2009) provided a method that well considers the effect of an external force on the momentum and momentum flux as well as the discrete lattice effect. In their method, the non-slip boundary condition at the boundary points is enforced. Wu and Shu (2010) applied this method to the simulation of particulate flows. Recently, Wang, Shu, Teo, and Wu (2015) proposed an immersed boundary lattice Boltzmann flux solver (IB-LBFS) for the simulation of two-dimensional fluid-structure interaction problems. The IB-LBFS effectively combines the advantages of the lattice Boltzmann flux solver in solving the flow field and the flexibility of the IBM in dealing with boundary conditions. The IB-LBFS presents a simple and effective approach of simulating complex fluid-structure problems on non-uniform grids.

In the case of multiple particles moving in fluids, collisions between particles need to be considered for the IB-LBM. The discrete element method (DEM), which was introduced by Cundall and Strack (1979), has been widely implemented for the numerical study of particle dynamics. The DEM can provide microstructural information for understanding the effect of particle shape on particle-particle contact. The DEM has been advanced for the modeling of non-spherical particles, such as ellipses, polygons, spheroids, cylinders, and polyhedra. Guo and Curtis (2015) reviewed the modeling of complex granular flows employing the DEM. Lu, Third, and Müller (2015) reviewed recent developments of the DEM in modeling particles of non-spherical shape. They reviewed advances in the formulation and implementation of non-spherical particle models, including advances in shape representation, algorithms for the efficient detection of contact, and the determination of contact parameters. For spherical particles, the normal contact force directed along the line of centers generates no torque. However, for non-spherical particles, the normal contact force generally does not pass through the center of gravity of the contacting particles and generates a torque. The determination of the contact point for circular or spherical particles is comparatively easy owing to the symmetry of the overlap between particles. However, a specific contact evaluation and detection technique is often used for non-circular or non-spherical particles. In particular, this technique may be cumbersome for three-dimensional polyhedral particles. Zhang et al. (2014) combined the IB-LBM model of Niu et al. (2006) and the DEM scheme to study the sedimentation of two-dimensional circular particles in incompressible Newtonian flows. However, the actual particles may have complex shape and we need to consider the shape effect during particle migration. 
At present, most numerical studies on particles moving in fluids focus on circular or spherical particles and there are few studies on non-circular or non-spherical particles. Recently, Galindo-Torres (2013) introduced a three-dimensional coupling law for the LBM and DEM to study problems involving the interaction between fluids and rigid structures. The DEM particles are modeled employing the sphero-polyhedra method, allowing the representation of general shapes, even non-convex shapes. However, in the work of Galindo-Torres, an empirically deduced weight coverage function was used for the coupling law of the LBM and DEM. In addition, as pointed out previously, the applied bounce-back boundary conditions may cause fluctuation of the forces and velocities of the particles.

The present study provides a two-dimensional lattice Boltzmann immersed boundary discrete element method (LB-IB-DEM) for multiple polygonal particles moving in fluids. This method is simple and easy to implement, and combines the advantages of the LBM, IBM, and DEM. In our coupling scheme, the IB-LBM of Niu et al. (2006) is used. Unlike in the work of Zhang et al. (2014), a DEM that can deal with the particle and wall shapes is used in our coupling scheme. Although each part of the proposed LB-IB-DEM is already known, the proposed coupling scheme is new, which can deal with the migration of particles having irregular geometries, and not only circular particles, in fluids. In addition, the incompressible LBM model of He-Luo (He \& Luo, 1997) and Gear's predictor-corrector algorithm of the DEM are used in our scheme, which also distinguishes our coupling scheme from the scheme of Zhang et al. (2014).

Although our method is two-dimensional at present, it can be straightforwardly extended to three-dimensional cases, in spite of this requiring a more complicated detection algorithm to be considered. The validity of the present method is demonstrated in the simulation of a single circular particle and two circular particles falling under gravity in incompressible viscous fluids. Through numerical simulations, we study the dynamics of particles and the characteristics of the flow field and discuss the effect of particle shape during the transport of polygonal particles.

\section{LB-IB-DEM}

\subsection{LB-IBM for fluid-particle interaction}


For the motion of polygonal particles in incompressible viscous fluids, a discrete lattice Boltzmann equation with a single-relaxation-time approximation is used to simulate the fluid flow:

$$
f_{\alpha}\left(\mathbf{r}+\mathbf{e}_{\alpha} \Delta t, t+\Delta t\right)-f_{\alpha}(\mathbf{r}, t)=-\frac{1}{\tau}\left[f_{\alpha}(\mathbf{r}, t)-f_{\alpha}^{\mathrm{eq}}(\mathbf{r}, t)\right]+F_{\alpha} \Delta t, \quad \alpha=1,2,3, \ldots, N,
$$

where $\mathbf{r}=(x, y)$ represents the spatial position, $\mathbf{e}_{\alpha}$ is the fluid particle velocity in the $\alpha$ direction, $N$ is the number of discrete fluid particle velocities, $\Delta t$ is the time step, $f_{\alpha}$ is the fluid particle distribution function, $\tau$ is the relaxation time, and $F_{\alpha}$ is the external force, which includes interaction forces of the particles acting on the fluid.

In this study, an incompressible D2Q9 lattice Boltzmann model proposed by He and Luo (1997) is used to simulate the fluid motion. This lattice Boltzmann model reduces the compressibility effects.

A momentum-exchange-based LB-IBM proposed by Niu et al. (2006) is used to simulate fluid-particle interactions and to obtain the force $\mathbf{F}_{\mathrm{T}}$ and torque $\mathbf{T}_{\mathrm{T}}$ of the fluid acting on particles. The LB-IBM uses a fixed Eulerian mesh for the fluid and Lagrangian points for solid boundaries, as shown in Fig. 1. In our study, a uniform Eulerian mesh is used. $h$ denotes the mesh spacing and $h=\delta_{x}=\delta_{y}$, where $\delta_{x}$ and $\delta_{y}$ are the mesh spacing in the $x$ - and $y$-directions, respectively. The LB-IBM used in our simulations was described in more detail by Niu et al. (2006).

Fig. 1

Considering the hydrodynamic force, gravitational force, and collision force, the governing equation of motion of solid particles can be written as

$$
\left\{\begin{array}{l}
m \mathbf{a}=m \mathbf{g}+\mathbf{F}_{\mathrm{T}}+\mathbf{F}_{\mathrm{c}} \\
I \frac{\partial^{2} \boldsymbol{\theta}}{\partial t^{2}}=\mathbf{T}_{\mathrm{T}}+\mathbf{M}_{\mathrm{c}}
\end{array},\right.
$$


where $m$ and $I$ are the mass and moment inertia of the solid particle, respectively. $\mathbf{F}_{\mathrm{c}}$ and $\mathbf{M}_{\mathrm{c}}$ are the contact force and torque, respectively. $\boldsymbol{\theta}$ is the angular position and $\mathbf{g}$ is the gravitational acceleration. $\mathbf{F}_{\mathrm{T}}$ and $\mathbf{T}_{\mathrm{T}}$ are the force and torque of the fluid acting on particles, respectively.

\subsection{DEM for polygonal particle motion}

This study adopts the DEM of Matuttis and Chen (2014) for polygonal particles. The detailed computation of $\mathbf{F}_{\mathrm{c}}$ and $\mathbf{M}_{\mathrm{c}}$ based on the polygon geometry was presented by Matuttis and Chen (2014).

The contact shape of two interacting polygons is shown in Fig. 2. The normal $\mathbf{n}$ is the normal of the line segment $S_{1} S_{2}$. The particle length factor $l$ is defined as

$$
l=\frac{4 r_{1} r_{2}}{r_{1}+r_{2}}
$$

where $r_{1}=\left\|\mathbf{r}_{1}\right\|$ and $r_{2}=\left\|\mathbf{r}_{2}\right\|, \mathbf{r}_{1}$ and $\mathbf{r}_{2}$ are vectors connecting the force point and the centers of mass $C_{1}$ and $C_{2}$.

The relative velocity $\mathbf{v}_{1,2}$ of the polygons at the contact point is

$$
\mathbf{v}_{1,2}=\mathbf{v}_{1}+\boldsymbol{\omega}_{1} \times \mathbf{r}_{1}-\left(\mathbf{v}_{2}+\boldsymbol{\omega}_{2} \times \mathbf{r}_{2}\right)
$$

where $\mathbf{v}_{1}$ and $\mathbf{v}_{2}$ are the velocities of the centers of mass, $\boldsymbol{\omega}_{1}$ and $\boldsymbol{\omega}_{2}$ are the angular velocities of the centers of mass.

Fig. 2

The contact force acting on the force point $P$ is

$$
\mathbf{F}_{\mathrm{c}}=\mathbf{F}_{\mathrm{ela}}+\mathbf{F}_{\mathrm{dam}}+\mathbf{F}_{\mathrm{fri}}+\mathbf{F}_{\mathrm{coh}}
$$


where $\mathbf{F}_{\text {ela }}$ is the elastic force, $\mathbf{F}_{\text {dam }}$ is the damping, $\mathbf{F}_{\text {fri }}$ is the friction, and $\mathbf{F}_{\text {coh }}$ is the cohesive force. The torque acting on polygons is

$$
\begin{aligned}
& \mathbf{M}_{\mathrm{c} 1}=\mathbf{r}_{1} \times \mathbf{F}_{\mathrm{c}}, \\
& \mathbf{M}_{\mathrm{c} 2}=\mathbf{r}_{2} \times\left(-\mathbf{F}_{\mathrm{c}}\right) .
\end{aligned}
$$

The magnitude of the elastic force is proportional to the area $A$ of overlap between the two particles:

$$
\left|F_{\text {ela }}\right|=\frac{E A}{l},
$$

where $E$ is the two-dimensional Young's modulus of particles, which has the units $\mathrm{N} / \mathrm{m}$. To obtain the area of overlap, the intersection points of the two interacting polygonal particles need to be computed. For example, we assume that there are only two intersection points $S_{1}$ and $S_{2}$, as shown in Fig. 3(a). To obtain the overlap area of $A J S_{2} K D S_{1}$, we need to know the positions of $S_{1}$ and $S_{2}$. We take $S_{1}$ as an example. $S_{1}$ is the intersection point of two lines, $A B$ and $C D$, as shown in Fig. 3(b). We define the ratio of the area $A_{2}=|\overrightarrow{A C} \times \overrightarrow{C D}|$ to the area $A_{1}=|\overrightarrow{A B} \times \overrightarrow{C D}|$ as $\lambda=A_{2} / A_{1}$. We then have $\overrightarrow{A S_{1}}=\lambda \overrightarrow{A B}$ and the position of $S_{1}$ is obtained. With the same process, we can obtain the position of $S_{2}$. After knowing the positions of intersection points, the area of overlap formed by the intersection points $\left(S_{1}, S_{2}\right)$ and corners $(A, D, J, K)$ that penetrate from one polygon into the other can be calculated.

Fig. 3

The normal damping is chosen to be

$$
F_{\mathrm{dam}}=-D \sqrt{E m^{*}} \frac{A(t-\delta t)-A(t)}{l \delta t},
$$


where $D$ is the damping coefficient and $m^{*}=\frac{m_{1} m_{2}}{m_{1}+m_{2}}$ is the normal reduced mass of the contacting particles.

The friction is treated as a modified Cundall-Strack model:

$$
\mathbf{F}_{\mathrm{t}}(t)=\mathbf{F}_{\mathrm{t}}(t-\delta t)-k_{\mathrm{t}, 1} \mathbf{v}_{\mathrm{t}} \delta t-k_{\mathrm{t}, 2} \mathbf{v}_{\mathrm{t}},
$$

where $\delta t$ is the time step of the DEM, $\mathbf{v}_{\mathrm{t}}$ is the tangential velocity, and the direction of $\mathbf{F}_{\mathrm{t}}$ is opposite that of $\mathbf{v}_{\mathrm{t}} \cdot k_{\mathrm{t}, 1}$ is the 'tangential stiffness' and $k_{\mathrm{t}, 2} \mathbf{v}_{\mathrm{t}}$ is a damping term that reduces the oscillations of $\mathbf{F}_{\mathrm{t}}$. In this study, we chose $k_{\mathrm{t}, 1}=2 E / 7$, which is exact for the contact of two spherical particles. $k_{\mathrm{t}, 2}$ is chosen as $k_{\mathrm{t}, 2}=\sqrt{k_{\mathrm{t}, 1} m_{\mathrm{t}}^{*}} / 4$, and $m_{\mathrm{t}}^{*}=m_{1} m_{2} /\left(m_{1}+m_{2}+m_{1} r_{1}^{2} / I_{1}+m_{2} r_{2}^{2} / I_{2}\right)$ is the tangential reduced mass, where $I_{1}$ and $I_{2}$ are the moments of inertia of the interacting particles. If the resulting tangential force exceeds the product of the normal force and the friction coefficient, it is truncated with the maximal friction value

$$
\mathbf{F}_{\mathrm{t}}(t)=\operatorname{sgn}\left(\mathbf{F}_{\mathrm{t}}(t)\right) \mu_{\mathrm{f}}\left\|\mathbf{F}_{\mathrm{n}}(t)\right\| \text {, if }\left\|\mathbf{F}_{\mathrm{t}}(t)\right\|>\mu_{\mathrm{f}}\left\|\mathbf{F}_{\mathrm{n}}(t)\right\|,
$$

where $\mathbf{F}_{\mathrm{n}}(t)$ is the normal force and $\mu_{\mathrm{f}}$ is the friction coefficient.

The direction of the cohesive force is the direction of the normal $\mathbf{n}$ and the magnitude of the cohesive force can be written as

$$
\left|F_{\text {coh }}\right|=E_{\text {coh }} l_{\text {coh }}
$$

where $E_{\text {coh }}$ is the cohesion coefficient as a fraction of $E, l_{\text {coh }}$ is the contact length and is chosen as the direct distance between the intersection points $S_{1}$ and $S_{2}$. 
In this study, we use Gear's second-order predictor-corrector algorithm to solve Eq. (2). This algorithm has two steps, the predictor step and the corrector step. The position of the polygon is $r_{0}$, the time step of the

DEM is $\delta t$, and we then write

$$
r_{0}(t+\delta t)=r_{0}(t)+\left(\frac{d r_{0}}{d t}\right) \delta t+\frac{(\delta t)^{2}}{2 !}\left(\frac{d^{2} r_{0}}{d t^{2}}\right)+\frac{(\delta t)^{3}}{3 !}\left(\frac{d^{3} r_{0}}{d t^{3}}\right)+\ldots
$$

We define $r_{1}=\left(\frac{d r_{0}}{d t}\right) \delta t, r_{2}=\frac{(\delta t)^{2}}{2 !}\left(\frac{d^{2} r_{0}}{d t^{2}}\right), r_{3}=\frac{(\delta t)^{3}}{3 !}\left(\frac{d^{3} r_{0}}{d t^{3}}\right)$, and the predicted position can be written as

$$
\left(\begin{array}{c}
r_{0}^{\mathrm{p}}(t+\delta t) \\
r_{1}^{\mathrm{p}}(t+\delta t) \\
r_{2}^{\mathrm{p}}(t+\delta t)
\end{array}\right)=\left(\begin{array}{lll}
1 & 1 & 1 \\
0 & 1 & 2 \\
0 & 0 & 1
\end{array}\right)\left(\begin{array}{c}
r_{0}(t) \\
r_{1}(t) \\
r_{2}(t)
\end{array}\right) .
$$

The actual forces $F$ acting on the particle, including particle-particle, particle-wall, and body forces, are calculated at the predicted particle position. The corrected position can then be written as

$$
\left(\begin{array}{c}
r_{0}^{\mathrm{c}}(t+\delta t) \\
r_{1}^{\mathrm{c}}(t+\delta t) \\
r_{2}^{\mathrm{c}}(t+\delta t)
\end{array}\right)=\left(\begin{array}{c}
r_{0}^{\mathrm{p}}(t+\delta t) \\
r_{1}^{\mathrm{p}}(t+\delta t) \\
r_{2}^{\mathrm{p}}(t+\delta t)
\end{array}\right)+\left(\begin{array}{l}
0 \\
1 \\
1
\end{array}\right) \Delta r
$$

where $\Delta r=r_{2}^{\mathrm{c}}-r_{2}^{\mathrm{p}}=\frac{F\left(r_{0}^{\mathrm{p}}\right)}{m}-r_{2}^{\mathrm{p}}$

\subsection{Computational sequence and parameters}

The computational sequence is illustrated in Fig. 4. In the present study, the time step $\Delta t$ of the LBM is equal to the mesh spacing $h$. The characteristic oscillation frequency of two particles in contact is $\omega=\sqrt{E / m^{*}}$. The period is then $T=1 /\left(2 \pi \sqrt{m^{*} / E}\right)$. To make the DEM stable, we chose the time step of the DEM $\delta t<1 /\left(2 \pi \sqrt{m_{\min } / E}\right)$, in which $m_{\min }$ is the minimal mass of the particles. In our study, $\Delta t>\delta t$ and several DEM time steps are carried out in one LBM time step to make the total time 
of the DEM equal to the total time of the LBM. To make the time step $\delta t$ of the DEM large in all our simulations and thus reduce the amount of calculation, a low Young's modulus of $10^{6} \mathrm{~N} / \mathrm{m}$ is chosen; this will result in the soft collisions of particles. We treat walls as fixed particles with density higher than that of moving particles. In our simulation, the cohesive force is not considered, thus simplifying the collision model. The gravitational acceleration $g=9.8 \mathrm{~m} / \mathrm{s}^{2}$ is used in all our simulations.

Fig. 4

\section{Results and discussion}

\subsection{Single particle in an incompressible viscous fluid}

To validate the code, we consider the motion of a single circular particle falling in an incompressible viscous fluid, as shown in Fig. 5. The fluid domain is a rectangular box with a width of $2 \mathrm{~cm}$ in the $x$-direction and a height of $6 \mathrm{~cm}$ in the $y$-direction. The viscosity of the fluid $\mu$ is $0.1 \mathrm{~g} /(\mathrm{cm} \mathrm{s})$. The density

of the fluid $\rho_{\mathrm{f}}$ is $1.0 \mathrm{~g} / \mathrm{cm}^{3}$ and the density of the particle $\rho_{\mathrm{p}}$ is $1.25 \mathrm{~g} / \mathrm{cm}^{3}$. The radius of the particle $r_{\mathrm{p}}$ is $0.125 \mathrm{~cm}$. Initially, the particle is statically set at $(1 \mathrm{~cm}, 4 \mathrm{~cm})$. The particle then falls under the effect of gravity. In the simulation, we use a uniform $201 \times 601$ mesh for the fluid domain and 60 uniformly distributed Lagrangian points to represent the boundary of the particle. These Lagrangian points are also the corners of the regular 60-sided polygon used to approximate the circumcircle. The friction coefficient is 0.3 and the damping coefficient is 1.5 . To allow a comparison with the results of previous studies, the particle Reynolds number and translational kinetic energy are defined as

$$
R e_{\mathrm{p}}=\frac{\rho_{\mathrm{p}} d_{\mathrm{p}} \sqrt{u_{\mathrm{p}}^{2}+v_{\mathrm{p}}^{2}}}{\mu}, E_{\mathrm{t}}=0.5 M\left(u_{\mathrm{p}}^{2}+v_{\mathrm{p}}^{2}\right),
$$

where $u_{\mathrm{p}}$ and $v_{\mathrm{p}}$ are the $x$-direction component and $y$-direction component of the velocity at the center point of the particle, respectively. $d_{\mathrm{p}}$ is the particle diameter and $M$ is the particle mass.

Fig. 5 
The $y$-coordinate of the particle center, $y$-direction component of the velocity of the particle center, particle Reynolds number, and translational kinetic energy are shown in Fig. 6. Before the particle hits the bottom wall, our simulation results agree well with the results for a circular particle obtained by Wan and Turek (2006). When the particle collides with the bottom wall, the difference is due to different collision parameters and models for our method and that used by Wan and Turek (2006). In our simulation, the energy is gradually consumed as the particle strikes the wall owing to the damping of the collision model. At time $t=1 \mathrm{~s}$, the particle remains on the wall with no vertical velocity after the impact in the case of our method while the particle still has a small vertical component of velocity in the simulation of Wan and Turek (2006) as seen in Fig. 6(b), which shows that our collision model is more physical.

Fig. 6

\subsection{Two particles in an incompressible viscous fluid}

To demonstrate the effect of the particle shape on particle-particle collision, we consider two particles falling under the effect of gravity in an incompressible viscous fluid, as shown in Fig. 7. The fluid domain is a box with width of $2 \mathrm{~cm}$ and height of $8 \mathrm{~cm}$. The fluid has viscosity $\mu$ of $0.01 \mathrm{~g} /(\mathrm{cm} \mathrm{s})$ and density $\rho_{\mathrm{f}}$ of $1.0 \mathrm{~g} / \mathrm{cm}^{3}$. The two particles have the same properties. The particle density $\rho_{\mathrm{p}}$ is $1.01 \mathrm{~g} / \mathrm{cm}^{3}$ and the particle radius is $0.1 \mathrm{~cm}$. In our simulation, to reduce the sensitivity of numerical disturbance, the first particle (P1) is initially statically set at $(0.996 \mathrm{~cm}, 7.2 \mathrm{~cm})$, not at $(0.999 \mathrm{~cm}, 7.2 \mathrm{~cm})$ as for Feng and Michaelides (2004), and the second particle $(\mathrm{P} 2)$ is set at $(1 \mathrm{~cm}, 6.8 \mathrm{~cm})$. We use a uniform $201 \times 801$ mesh for the fluid domain and 60 uniformly distributed Lagrangian points to represent the boundary of each particle. A friction coefficient of 0.04 and damping coefficient of 0.5 are used for this problem.

Fig. 7

The simulation results of particle positions and velocities are shown in Fig. 8. This problem is the so-called drafting-kissing-tumbling problem, presented in Fortes, Joseph, and Lundgren (1987). In the drafting stage, the two particles attract each other owing to the low pressure. When the two particles are within a certain separation, they repeatedly collide in the kissing stage. Afterward, they roll on each other 
and separate in the tumbling stage. As pointed out by Patankar (2001) this tumbling process is a realization of an instability stage and can be affected by the accuracy of the solution procedure and the modeling of the collision force. Owing to the polygon approximation of a circle and different collision models, there is a difference between our results and the results obtained by Feng and Michaelides (2004) after the drafting stage. Fig. 8 also shows a difference between the results obtained by Zhang et al. (2014) and those obtained by Feng and Michaelides (2004). It is noteworthy that, through the use of our collision model, a fluctuation of the $y$-direction velocity in the kissing stage is observed in our simulation.

Fig. 8

To investigate the effect of the grid size, we compare the results of the longitudinal velocities for three computational grids, as shown in Fig. 9. The results are almost identical for a coarser $200 \times 800$ grid and a finer $300 \times 1200$ grid before the tumbling process. The results for increasing grid resolution thus converge before the tumbling process. Even though the tumbling process is unstable, good qualitative and quantitative agreement between the results obtained with coarse and fine grids can be observed in Fig. 9 .

\section{Fig. 9}

To compare the dynamics of polygonal particles with the dynamics of circular particles, we simulate two regular polygons falling in the same fluid. To reduce the sensitivity of numerical disturbance, the first particle is initially set at $(0.99 \mathrm{~cm}, 7.2 \mathrm{~cm})$. The radius of the circumcircle of polygon is equal to the radius of the circular particle and other parameters are kept unchanged. The first corner of the particle lies on a ray that starts from the center of mass of the particle and is along the $x$-direction. We number the corners in the counterclockwise direction. The positions and velocities of regular triangular particles (three edges), regular hexagonal particles (six edges), and circular particles (60 edges) are shown in Fig. 10.

Fig. 10

The particle shape affects not only the drag but also the collision modes between particles. Triangular particles and hexagonal particles fall more slowly than circular particles, which can be seen from the vertical positions of particles, as shown in Fig. 10(a). Additionally, owing to their edges and corners, the 
behaviors of hexagonal particles in the kissing stage (after approximately $t=1.5 \mathrm{~s}$ ) and tumbling stage (after approximately $t=2.5 \mathrm{~s}$ ) are different from those of circular particles. Compared with the case for circular particles, hexagonal particles may undergo corner-edge, edge-edge, or corner-corner collisions in the kissing stage, leading to larger moments of rotation. The horizontal velocities of hexagonal particles sharply fluctuate after the particles enter the kissing stage (at approximately $t=1.5 \mathrm{~s}$ ), which reflects that intense collisions may occur. Circular particles can slide along the contact area easily and collisions between two circular particles in the kissing stage are gentle. The centers of two particles start to slowly deviate from the channel center and to move closer. When the particles completely enter the tumbling stage (at approximately $t=2.5 \mathrm{~s}$ ), the separation of hexagonal particles is more gradual than that of circular particles owing to the edge-edge contact of hexagonal particles. The horizontal separation of the centers of hexagonal particles is less than that of circular particles, as seen in Fig. 10(b).

It is noteworthy that triangular particles did not come into contact with each other in our simulations. The distance between the two triangular particles was always greater than the diameter of the circumcircle, as seen in Fig. 11. Fig. 11 also shows that the trajectories of the regular triangular (three edges), square (four edges), and pentagonal (five edges) particles, which deviated to the left domain of the fluid, are different from those of the regular hexagonal (six edges) and circular (regular 60-sided polygon, 60 edges) particles, which deviated to the right domain of the fluid. The positions of two polygonal particles at different stages are shown in Fig. 12. We can see the difference in migration pattern between polygonal particles and circular particles.

Figs. $11 \& 12$

The contours of vorticity for polygonal particles falling under gravity in the kissing stage at $t=2.0 \mathrm{~s}$ are shown in Fig. 13. We see that a large stretched clockwise vortex attaches to the left side of particles while a large anticlockwise vortex attaches to the right side for both polygonal and circular particles. However, for polygonal particles, because of their corners, small vortexes are generated near their corners and these vortexes can create a torque for rotation of the particles. Owing to the effects of finite edges and corners, vortex patterns of polygonal particles are different from those of circular particles. The vortex 
patterns affect the flow field and lead to a difference in particle motions between polygonal particles and circular particles.

Our simulation results demonstrate that the shape of particles can make non-circular particles behave differently from circular particles and suggest that we should consider the geometry of the particle shape for particle motion in fluids. The present numerical method provides a way of understanding the mechanics of non-circular particles moving in fluids.

Fig. 13

\subsection{Multiple particles in an incompressible viscous fluid}

To demonstrate that our method is capable of handling the sedimentation of multiple particles, a simulation is carried out for the sedimentation of 16 polygonal particles in an incompressible viscous fluid. The fluid domain is a square box with width of $2 \mathrm{~cm}$ and height of $2 \mathrm{~cm}$. The viscosity of the fluid $\mu$ is 0.1 $\mathrm{g} /(\mathrm{cm} \mathrm{s})$, the density of the fluid $\rho_{\mathrm{f}}$ is $1.0 \mathrm{~g} / \mathrm{cm}^{3}$ and the density of the particle $\rho_{\mathrm{p}}$ is $2.0 \mathrm{~g} / \mathrm{cm}^{3}$. For regular polygons, the circumcircle diameter of particles $d_{\mathrm{p}}$ is $0.325 \mathrm{~cm}$. We use a uniform $201 \times 201$ mesh for the fluid domain and 120 Lagrangian points to represent the boundary of each particle. A friction coefficient of 0.3 and a damping coefficient of 1.5 are used in this simulation.

Polygonal particles are initially statically positioned in the fluid domain. The first corner of a particle lies on a ray that starts from the center of mass of the particle and is along the $x$-direction. We number the corners in the counterclockwise direction. The distance between the center of the top-left particle and the left or top fluid boundary is $2.875 d_{\mathrm{p}}$. We first let the distance between the centers of any two neighboring particles be $0.875 d_{\mathrm{p}}$. We then move particles in the even (look from top to down) lines $0.125 d_{\mathrm{p}}$ along the horizontal direction. The initial positions of particles can be seen in Fig. 14.

The simulation results for the sedimentation of 16 particles in an incompressible viscous fluid at different time stages are also shown in Fig. 14. Two vortexes form in the fluid domain when the particles fall to the wall below. The left vortex is clockwise while the right vortex is anticlockwise. These two 
vortexes spread particles to the left and right walls. When particles fall, they stack up at the bottom-left and bottom-right corners of the fluid domain. Compared with circular particles (regular 120-sided polygons), regular pentagons and regular hexagons can stack up more easily in the vertical direction because of their corners and edges. The circular particles can easily slide along their contact line and they are finally almost uniformly distributed on the wall below. For our simulation parameters, the results show that regular hexagons stay in the fluid more steadily than regular pentagons possibly owing to their centrosymmetric shape.

Fig. 14

To further demonstrate that our method can deal with particle shapes that are more general, we simulate the sedimentation of 16 irregular polygons in fluids. The initial centers of the irregular polygons are the same as those of the regular polygons. However, the particle shapes deviate from regular polygons. The number of corners of each particle is generated randomly between four and eight. The corner positions of each particle deviate from those of regular polygons with random variations. The circumcircle diameter of particles is approximately $0.3 \mathrm{~cm}$. The sedimentation of 16 irregular polygons in an incompressible viscous fluid at different time stages is shown in Fig. 15. Fig. 15 also reveals two vortexes generated in the fluid domain. Comparing Figs. 14 and 15, we see that the stacking structure of irregular polygons is different from that of circles and similar to that of regular hexagons.

Fig. 15

To quantitatively describe the dispersion of particles, we use the standard deviation of the particle positions in the $\mathrm{x}$-direction. The standard deviation $\sigma$ is defined as

$$
\sigma=\sqrt{\frac{1}{n-1} \sum_{i=1}^{n}\left(x_{i}-\bar{x}\right)^{2}},
$$


where $n=16$ is the total number of particles and $\bar{x}=\sum_{i=1}^{16} x_{i}$. For regular polygonal particles, $x_{i}$ is the particle center position in the $x$-direction. For irregular polygonal particles, $x_{i}$ is the average position of corners in the $x$-direction.

The time histories of the standard deviation $\sigma$ for particles with different shapes are shown in Fig. 16. From Fig. 16, we see that the standard deviation $\sigma$ of circular particles is always largest before $t=0.25 \mathrm{~s}$, which indicates that circular particles are most distributed before $t=0.25 \mathrm{~s}$. Because circular particles can easily roll on the wall surface, they migrate closely to the corners of the fluid domain. However, it is difficult for polygonal particles to roll on the wall surface. After $t=0.25 \mathrm{~s}$, circular particles easily slide along the contact areas between two contact particles and move toward the middle of the wall, which leads to a more uniform distribution. Thus, $\sigma$ of circular particles is expected to decline, as shown in Fig. 16. However, polygonal particles hold relative stable structures and the changes in $\sigma$ are small after $t=0.25 \mathrm{~s}$. Compared with the case for hexagons, $\sigma$ of pentagons has a slight decline between $t=0.25$ and $0.35 \mathrm{~s}$, which indicates that hexagons hold structures that are more stable. In addition, we find that $\sigma$ of random polygons is between the values for hexagons and pentagons. When the particles remain on the surfaces, $\sigma$ is constant as time passes.

Fig. 16

\section{Conclusions}

A novel two-dimensional numerical method was presented for the simulation of polygonal particles falling in viscous incompressible fluids. The method combines the advantages of the LBM, IBM, and DEM, and is simple and easy to implement. The effects of the particle shape on particle-particle and particle-wall collisions were considered in our simulations. The presented method can handle the sedimentation of particles with irregular geometry in fluids. The sedimentation of a single particle and two circular particles was investigated by employing our method and the simulation results are in agreement with results reported in the literature. The dynamics of two regular polygonal particles falling in fluids were compared with those 
of circular particles. Finally, through the simulation of 16 particles moving in a viscous incompressible fluid, we demonstrated that our method can deal with multiple polygonal particles moving in fluids.

The simulation results showed that the particle shape affects not only the drag forces but also the modes of collision between particles when particles move in viscous incompressible fluids. Non-circular particles behave differently from circular particles. Polygonal particles can stack up more easily than circular particles in the vertical direction on the surface of the wall below in the fluid domain, while circular particles can more easily slide along their contact line and are finally almost uniformly distributed on the wall below. The present numerical method provides a way of understanding the mechanics of non-circular particles moving in viscous incompressible fluids.

Finally, we point out that, even though the present simulations are limited to two-dimensional cases, the equations and method are general and the presented LB-IB-DEM may be easily expanded to three-dimensional cases, despite more efforts being required for the development of the DEM of three-dimensional non-spherical particles.

\section{Acknowledgements}

This study was funded by the National Science Foundation of China (Grant No. 11272176).

\section{References}

Challabotla, N. R., Zhao, L., \& Andersson, H. I. (2015). Orientation and rotation of inertial disk particles in wall turbulence. Journal of Fluid Mechanics, 766, doi: 10.1017/jfm.2015.38.

Cundall, P. A., \& Strack, O. D. (1979). A discrete numerical model for granular assemblies. Geotechnique, $29(1), 47-65$.

Di, S., \& Ge, W. (2015). Simulation of dynamic fluid-solid interactions with an improved direct-forcing immersed boundary method. Particuology, 18, 22-34.

Feng, Z. G., \& Michaelides, E. E. (2004). The immersed boundary-lattice Boltzmann method for solving fluid-particles interaction problems. Journal of Computational Physics, 195(2), 602-628.

Fortes, A. F., Joseph, D. D., \& Lundgren, T. S. (1987). Nonlinear mechanics of fluidization of beds of spherical particles. Journal of Fluid Mechanics, 177, 467-483. 
Galindo-Torres, S. A. (2013). A coupled discrete element lattice boltzmann method for the simulation of fluid-solid interaction with particles of general shapes. Computer Methods in Applied Mechanics and Engineering, 265, 107-119.

Guo, Y., \& Curtis, J. S. (2015). Discrete element method simulations for complex granular flows. Annual Review of Fluid Mechanics, 47, 21-46.

Guo, Z., Zheng, C., \& Shi, B. (2002). Discrete lattice effects on the forcing term in the lattice Boltzmann method. Physical Review E, 65(4), 046308.

He, X., \& Luo, L. S. (1997). Lattice Boltzmann model for the incompressible Navier-Stokes equation. Journal of Statistical Physics, 88(3-4), 927-944.

Ladd, A. J. (1994a). Numerical simulations of particulate suspensions via a discretized Boltzmann equation. Part 1. Theoretical foundation. Journal of Fluid Mechanics, 271(1), 285-309.

Ladd, A. J. (1994b). Numerical simulations of particulate suspensions via a discretized Boltzmann equation. Part 2. Numerical results. Journal of Fluid Mechanics, 271, 311-339.

Ladd, A. J. C., \& Verberg, R. (2001). Lattice-Boltzmann simulations of particle-fluid suspensions. Journal of Statistical Physics, 104(5-6), 1191-1251.

Lu, G., Third, J. R., \& Müller, C. R. (2015). Discrete element models for non-spherical particle systems: From theoretical developments to applications. Chemical Engineering Science, 127, 425-465.

Matuttis, H. G., \& Chen, J. (2014). Understanding the discrete element method: Simulation of non-spherical particles for granular and multi-body systems. New York: John Wiley \& Sons.

Niu, X. D., Shu, C., Chew, Y. T., \& Peng, Y. (2006). A momentum exchange-based immersed boundary-lattice Boltzmann method for simulating incompressible viscous flows. Physics Letters A, 354(3), 173-182.

Noble, D. R., \& Torczynski, J. R. (1998). A lattice-Boltzmann method for partially saturated computational cells. International Journal of Modern Physics C, 9(8), 1189-1201.

Patankar, N. A. (2001). A formulation for fast computations of rigid particulate flows. Center for Turbulence Research Annual Research Briefs, 2001, 185-196.

Peskin, C. S. (1977). Numerical analysis of blood flow in the heart. Journal of Computational Physics, 25(3), $220-252$

Peskin, C. S. (2002). The immersed boundary method. Acta Numerica, 11, 479-517.

Shu, C., Liu, N., \& Chew, Y. T. (2007). A novel immersed boundary velocity correction-lattice Boltzmann method and its application to simulate flow past a circular cylinder. Journal of Computational Physics, 226(2), 1607-1622.

Uhlmann, M. (2005). An immersed boundary method with direct forcing for the simulation of particulate flows. Journal of Computational Physics, 209(2), 448-476. 
Voth, G. A. (2015). Disks aligned in a turbulent channel. Journal of Fluid Mechanics, 772, doi: 10.1017/jfm.2015.144.

Wan, D., \& Turek, S. (2006). Direct numerical simulation of particulate flow via multigrid FEM techniques and the fictitious boundary method. International Journal for Numerical Methods in Fluids, 51(5), $531-566$.

Wang, Y., Shu, C., Teo, C. J., \& Wu, J. (2015). An immersed boundary-lattice Boltzmann flux solver and its applications to fluid-structure interaction problems. Journal of Fluids and Structures, 54, 440-465.

Wu, J., \& Shu, C. (2009). Implicit velocity correction-based immersed boundary-lattice Boltzmann method and its applications. Journal of Computational Physics, 228(6), 1963-1979.

Wu, J., \& Shu, C. (2010). Particulate flow simulation via a boundary condition-enforced immersed boundary-lattice Boltzmann scheme. Communications in Computational Physics, 7(4), 793.

Xiong, Q., Li, B., Chen, F., Ma, J., Ge, W., \& Li, J. (2010). Direct numerical simulation of sub-grid structures in gas-solid flow-GPU implementation of macro-scale pseudo-particle modeling. Chemical Engineering Science, 65(19), 5356-5365.

Xiong, Q., Li, B., Xu, J., Wang, X., Wang, L., \& Ge, W. (2012a). Efficient 3D DNS of gas-solid flows on Fermi GPGPU. Computers \& Fluids, 70, 86-94.

Xiong, Q., Li, B., Zhou, G., Fang, X., Xu, J., \& Wang, J., et al. (2012b). Large-scale DNS of gas-solid flows on Mole-8.5. Chemical Engineering Science, 71, 422-430.

Yu, Z., \& Fan, L. S. (2010). Lattice Boltzmann method for simulating particle-fluid interactions. Particuology, 8(6), 539-543.

Zhang, H., Tan, Y., Shu, S., Niu, X., Trias, F. X., \& Yang, D., et al. (2014). Numerical investigation on the role of discrete element method in combined LBM-IBM-DEM modeling. Computers \& Fluids, 94, $37-48$. 


\title{
Numerical simulation of polygonal particles moving in incompressible viscous fluids
}

\author{
Xiaowu Fu ${ }^{\mathrm{a}}$, Zhaohui Yao ${ }^{\mathrm{a}, *}$, Xiwen Zhang ${ }^{\mathrm{a}}$
}

\section{Figure captions:}

Fig. 1. Schematic diagram of solid boundary points and fluid points.

Fig. 2. Schematic diagram of the contact shape of two interacting polygons. The centroid of the overlap area is the force point $P . S_{1}$ and $S_{2}$ are the intersection points of the outlines of the two polygons. $\mathbf{r}_{1}$ and $\mathbf{r}_{2}$ are vectors from the centers of mass $C_{1}$ and $C_{2}$ to $P$, respectively. The normal and tangential directions for the contact are $\mathbf{n}$ and $\mathbf{t}$ respectively. The angular velocities of the two polygons are $\boldsymbol{\omega}_{1}$ and $\boldsymbol{\omega}_{2}$, respectively.

Fig. 3. (a) Overlapping polygons with intersection points $\left(S_{1}, S_{2}\right)$ and corners $(A, D, J, K)$ that penetrate from one polygon into the other. The point $S_{1}$ lies on the edges $A B$ and $C D$. (b) The position of the intersection point $S_{1}$ is calculated using the vectors $\overrightarrow{A B}, \overrightarrow{C D}$, and $\overrightarrow{A C}$. Additionally, $\overrightarrow{A B}=\overrightarrow{E F}$, $\overrightarrow{C D}=\overrightarrow{A E}$, and $\overrightarrow{A C}=\overrightarrow{E D}$

Fig. 4. Computational sequence.

Fig. 5. Schematic diagram of a single particle falling in an incompressible viscous fluid.

Fig. 6. Time histories of a single particle falling down in an incompressible viscous fluid for: (a) $y$-coordinate of the particle center ${ }^{y_{\mathrm{p}}}$, (b) $y$-direction component of the particle velocity $v_{\mathrm{p}}$, (c) particle Reynolds number $R e_{\mathrm{p}}$, and (d) translational kinetic energy $E_{\mathrm{t}}$. 
Fig. 7. Schematic diagram of two particles falling in an incompressible viscous fluid.

Fig. 8. Time histories of two particles falling in an incompressible viscous fluid for: (a) $y$-coordinate of the particle centers $y_{\mathrm{p}}$, (b) $x$-coordinate of the particle centers ${ }^{x_{\mathrm{p}}}$, (c) $y$-direction component of the particle velocities $v_{\mathrm{p}}$, and (d) $x$-direction component of the particle velocities $u_{\mathrm{p}}$.

Fig. 9. Longitudinal velocities of particles obtained with different grids.

Fig. 10. Time histories of two regular polygonal particles falling in an incompressible viscous fluid, where E3 (three edges), E6, and E60 refer to regular triangles, regular hexagons, and regular 60-sided polygons, respectively, for: (a) $y$-coordinate of the particle centers $y_{\mathrm{p}}$, (b) $x$-coordinate of the particle centers $x_{\mathrm{p}}$, (c) $y$-direction component of the particle velocities $v_{\mathrm{p}}$, and (d) $x$-direction component of the particle velocities $u_{\mathrm{p}}$

Fig. 11. Trajectories (a) and separation (b) of the two polygonal particles in fall, where the critical distance is defined here as the diameter of the circular particle.

Fig. 12. Sedimentation of two polygonal particles in different time stages for: (a) regular triangle (three edges), (b) square (four edges), (c) regular pentagon (five edges), (d) regular hexagon (six edges), and (e) regular 60-sided polygon (60 edges).

Fig. 13. Contours of vorticity for polygonal particles falling under gravity in the kissing stage at $t=2 \mathrm{~s}$, where the color bar shows relative values and positive values represent anticlockwise vorticities while negative values represent clockwise vorticities, for: (a) square, (b) regular pentagon, (c) regular hexagon, and (d) regular 60-sided polygon.

Fig. 14. Sedimentation of 16 polygonal particles in an incompressible viscous fluid at different time stages, for: (a) regular pentagons, (b) regular hexagons, and (c) circles (regular 120-sided polygons).

Fig. 15. Sedimentation of 16 irregular polygons in an incompressible viscous fluid at different time stages.

Fig. 16. Time histories of the standard deviation $\sigma$ for particles of different shapes. 


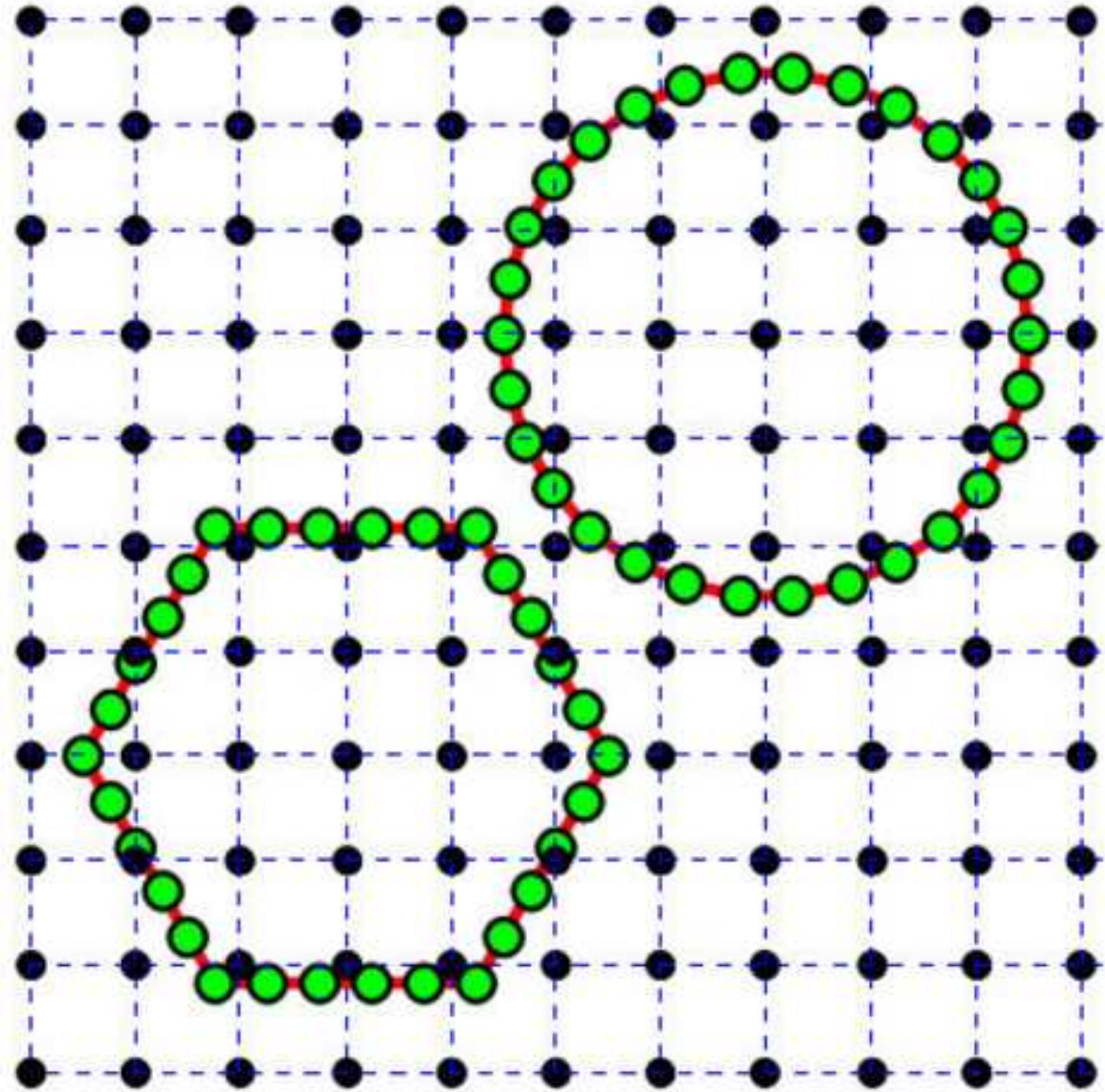

Solid boundaries $O$ Langranian points

- Eulerian points 


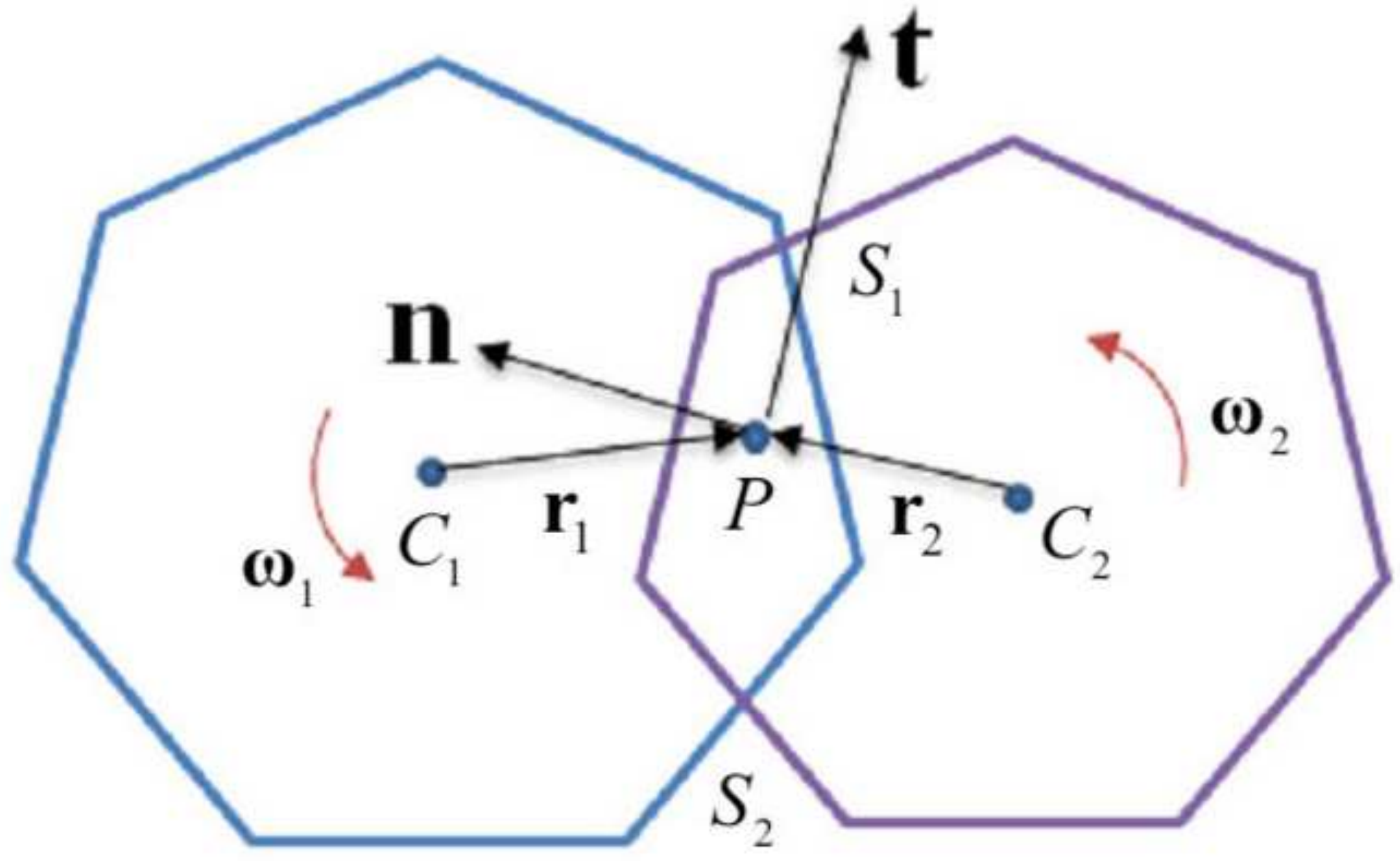



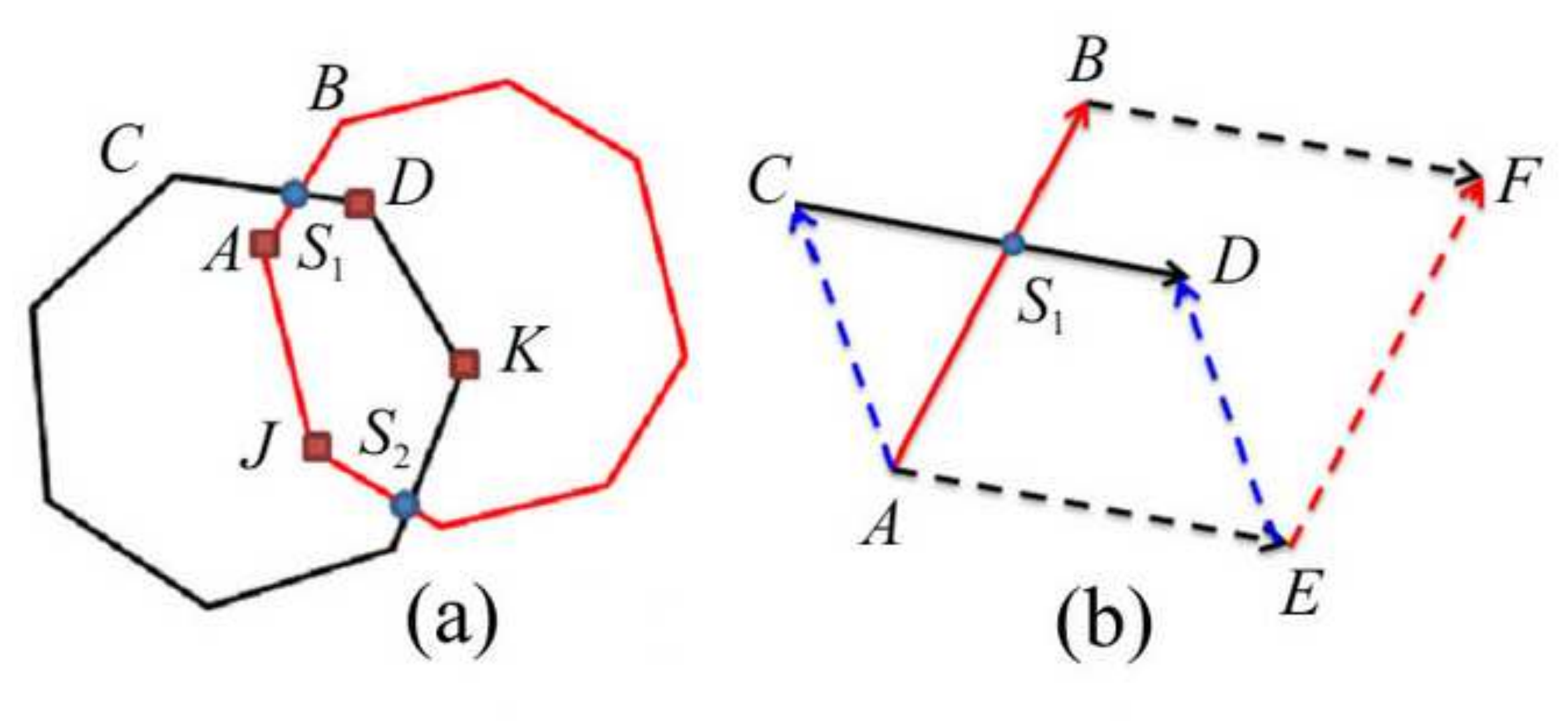

(b) $E$

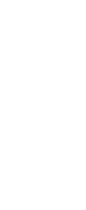

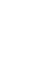

.




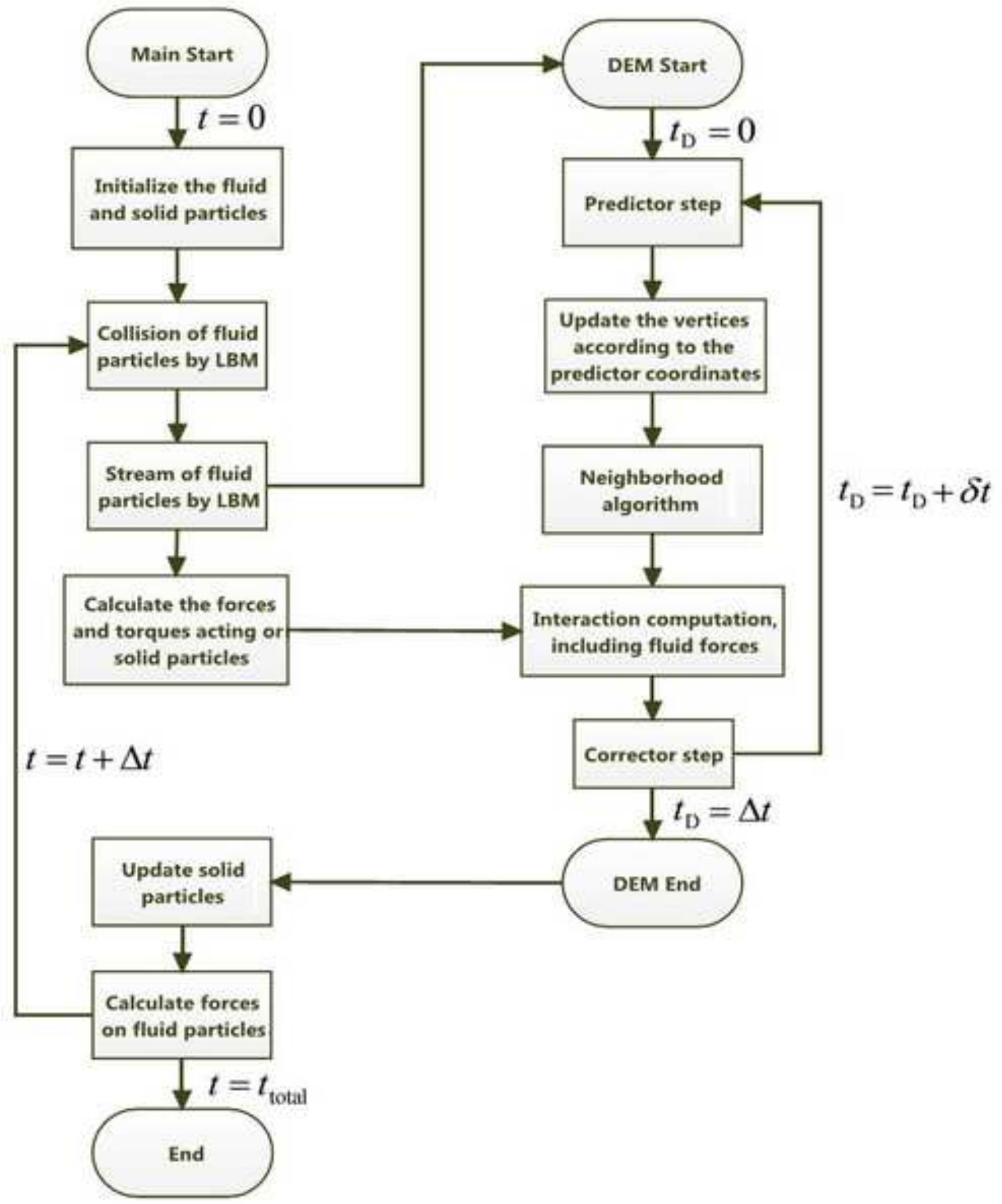




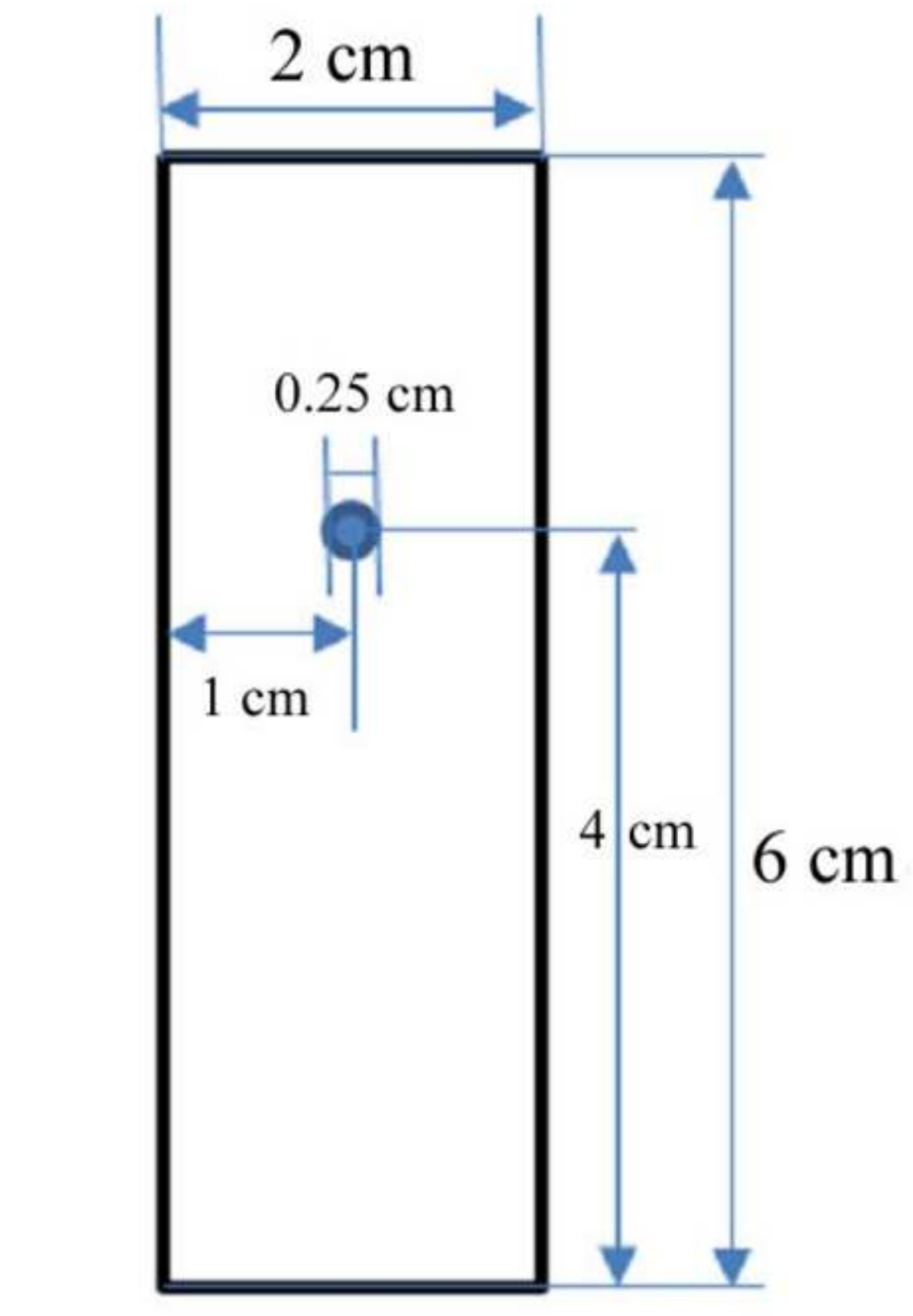

$\mathrm{g}$
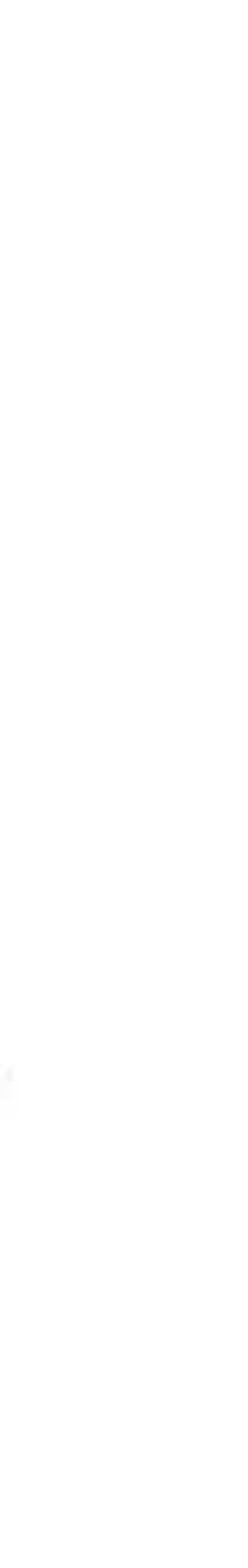

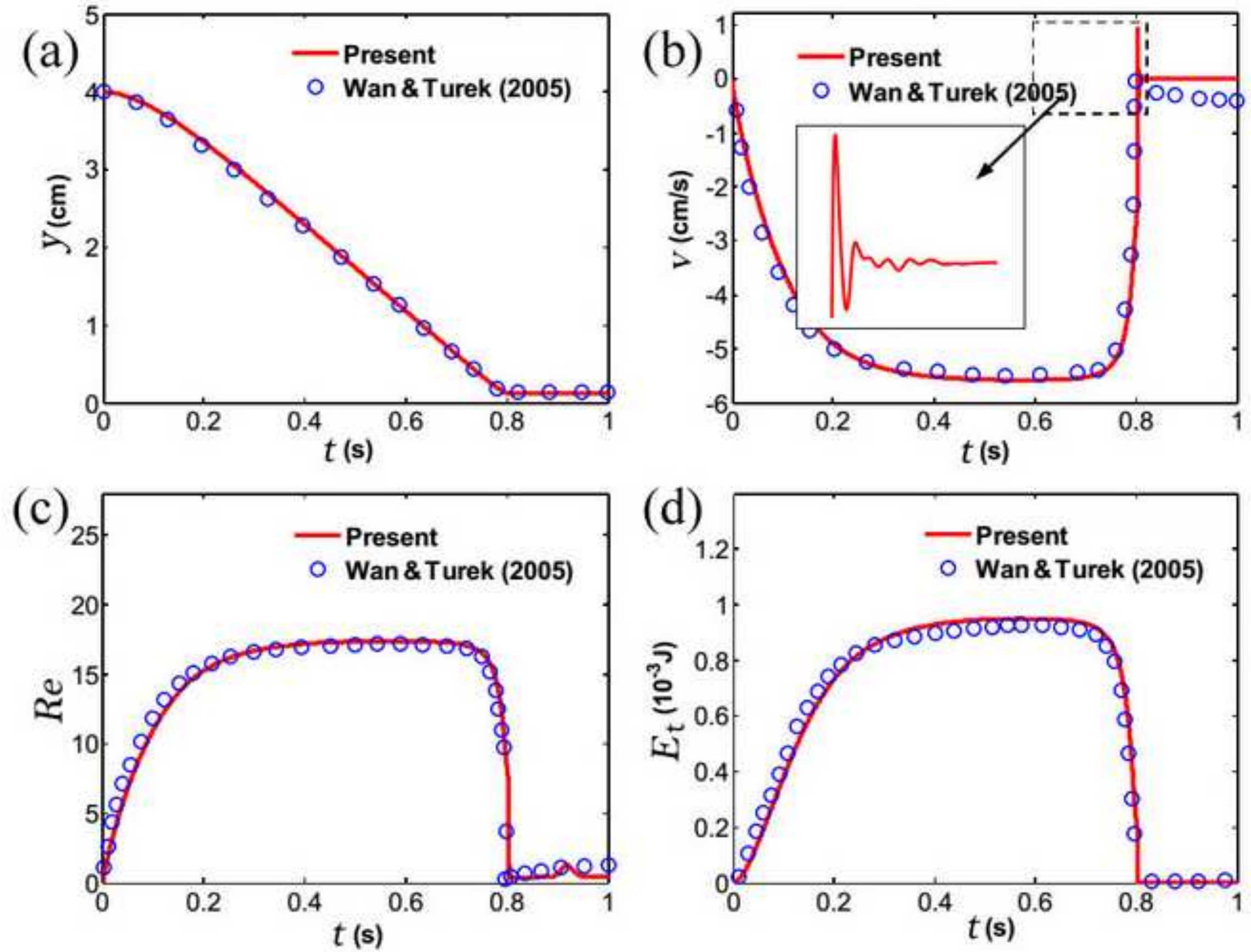


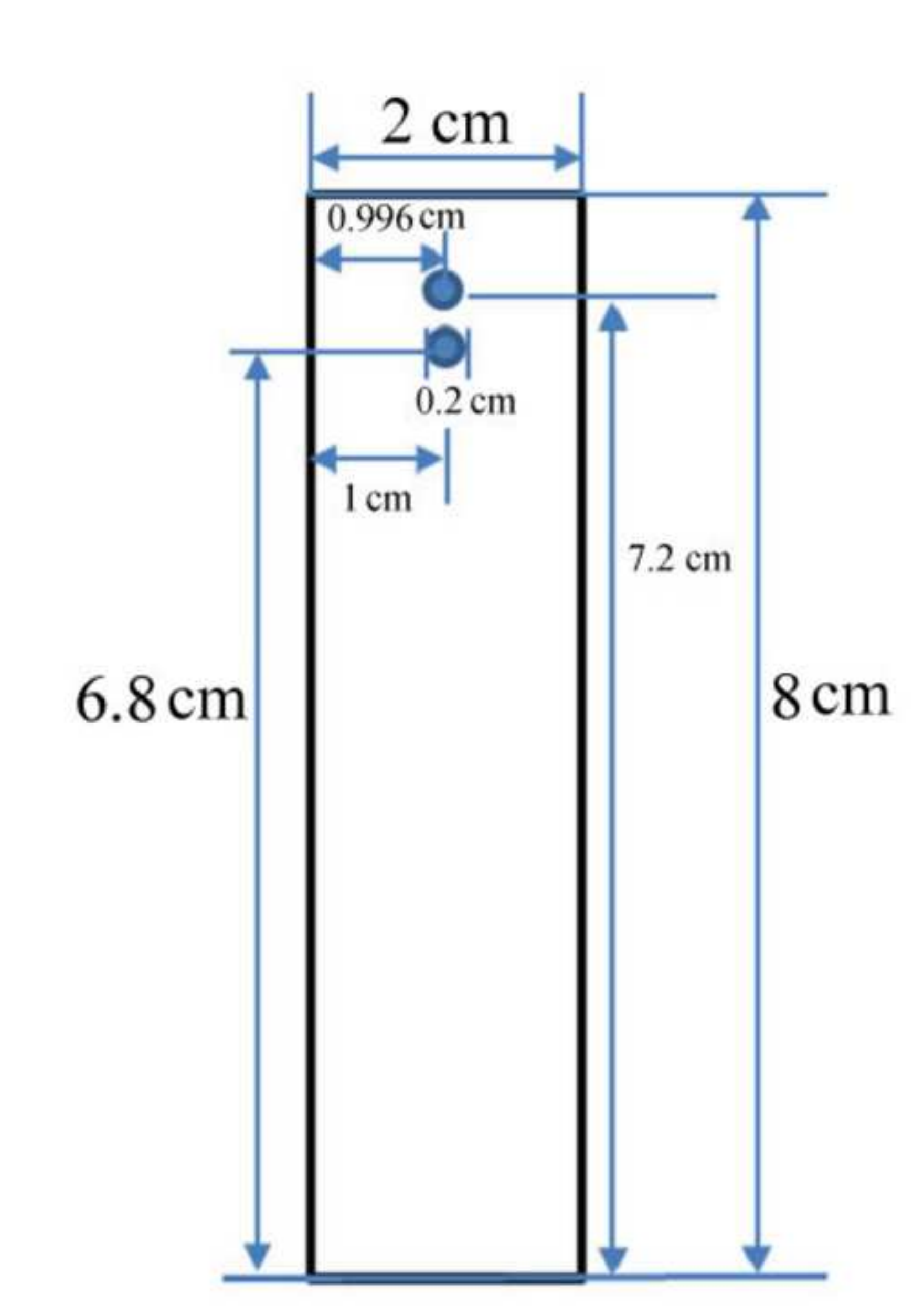

\section{.}

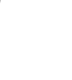



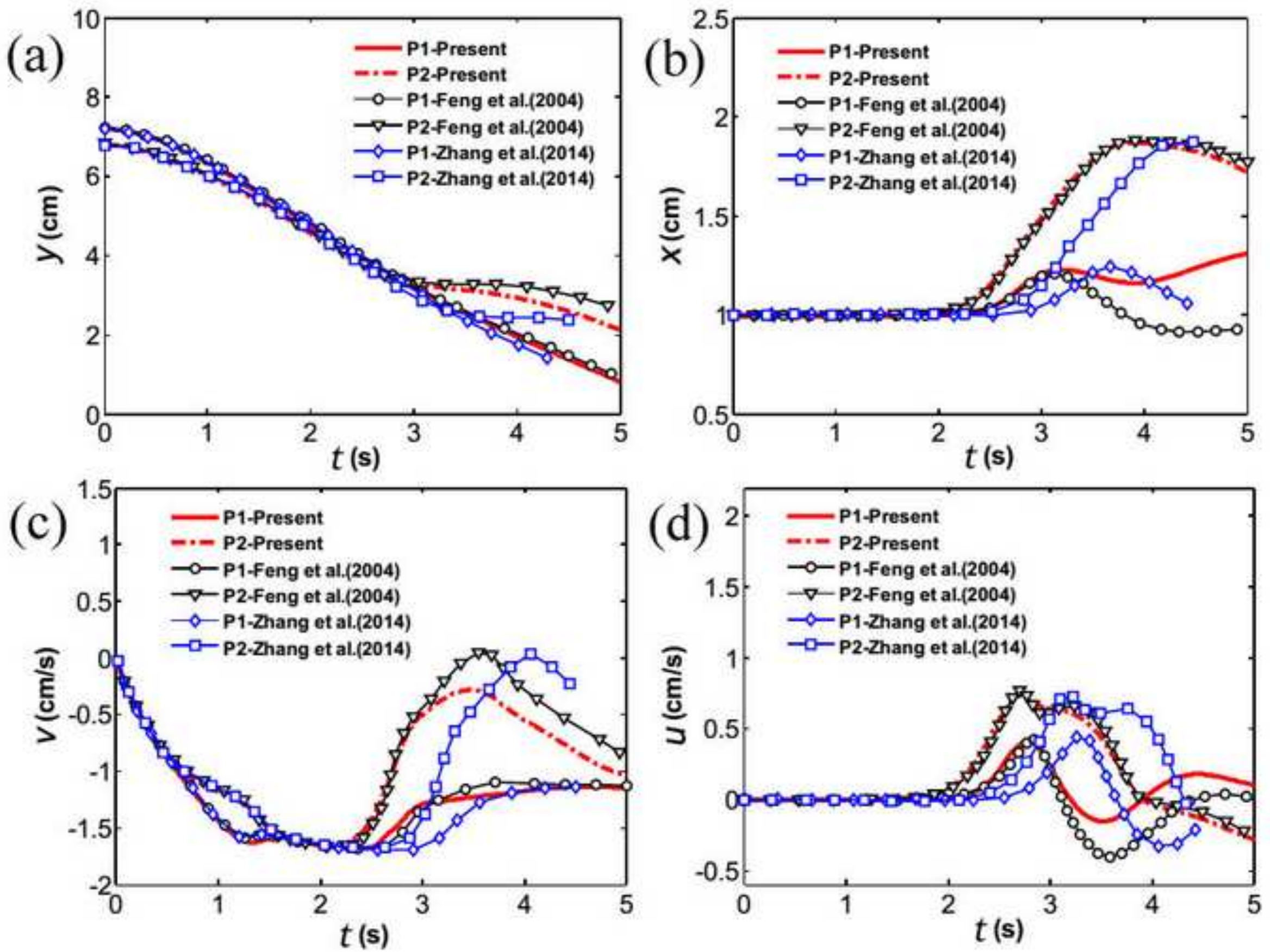


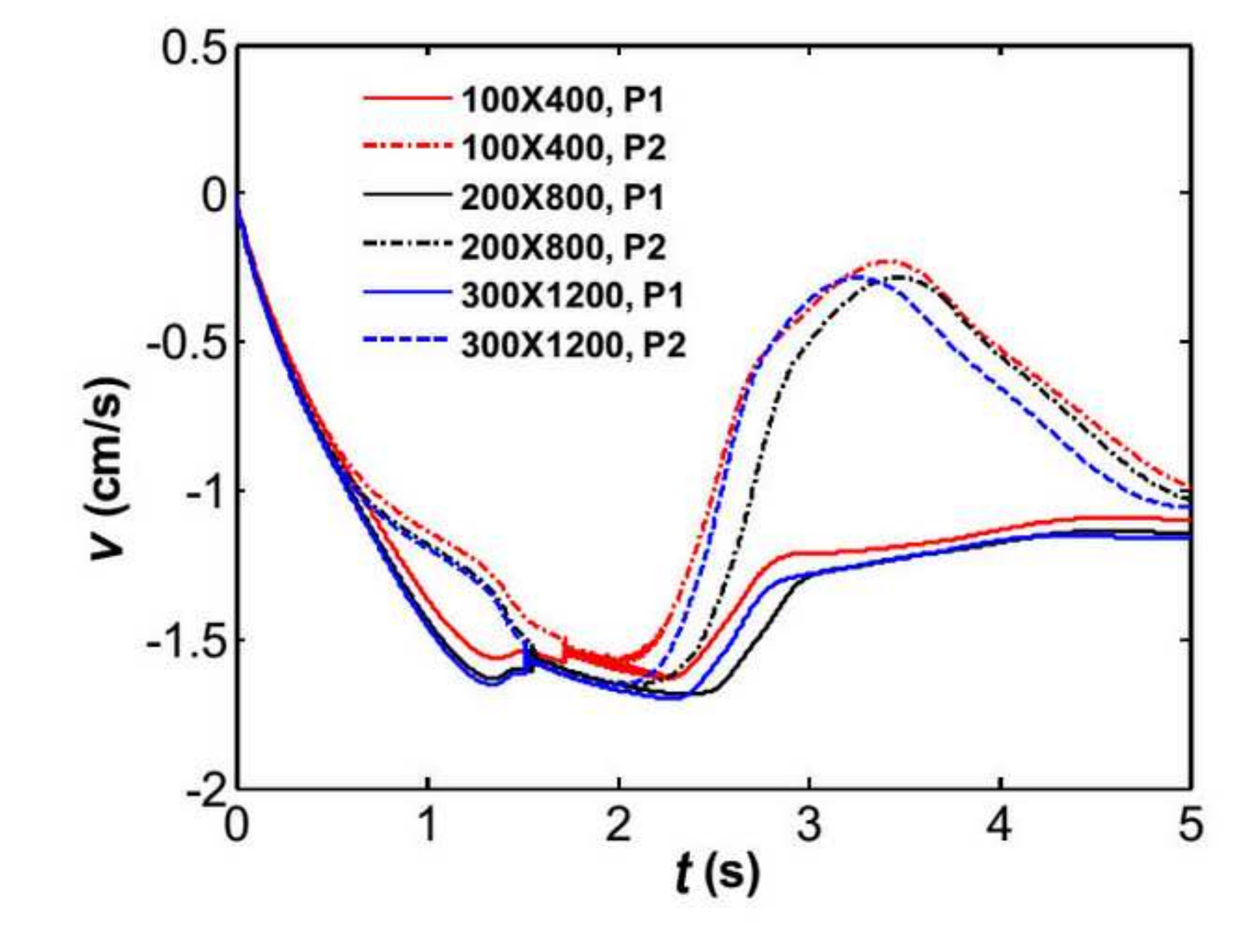

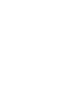

.

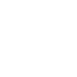

.



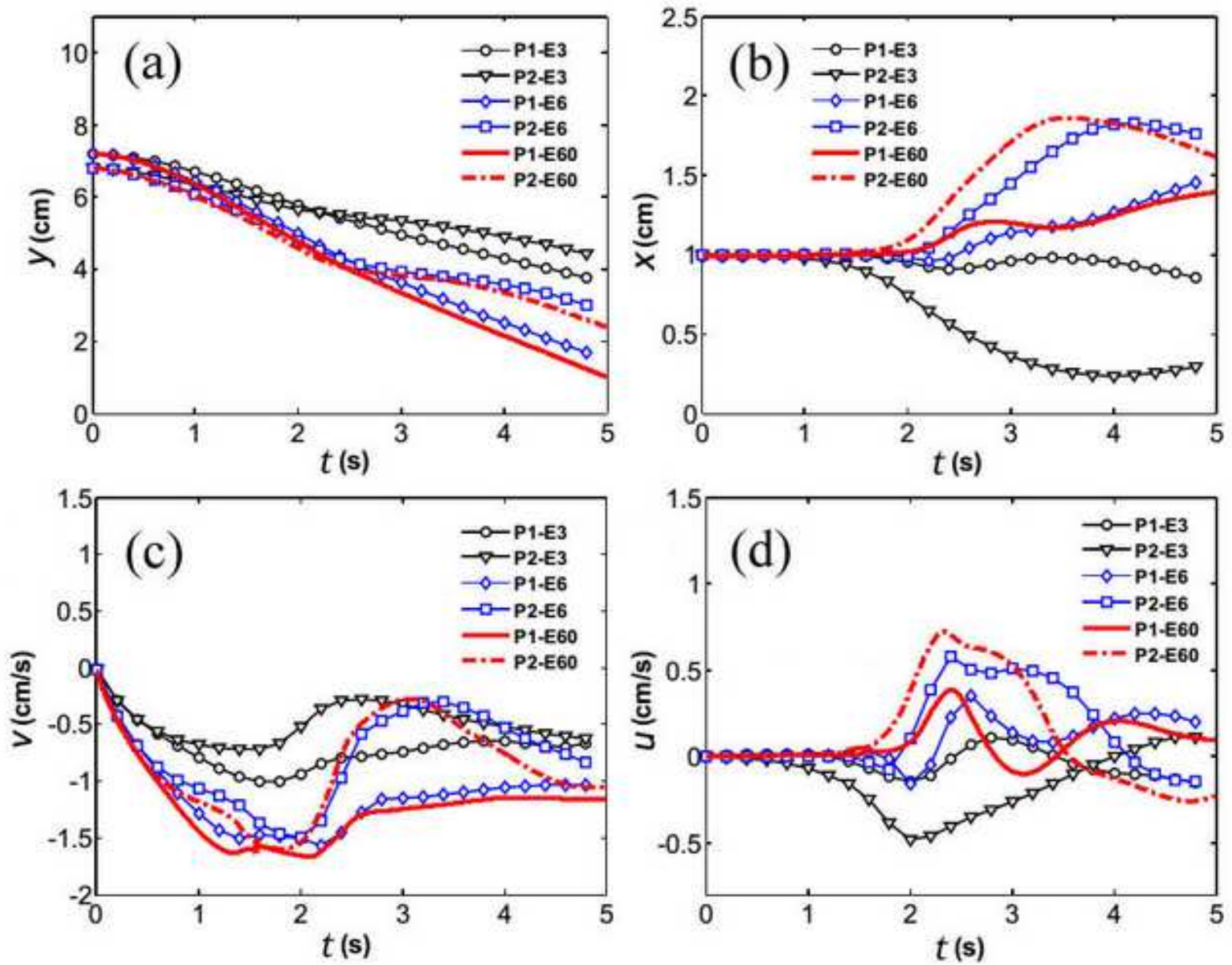

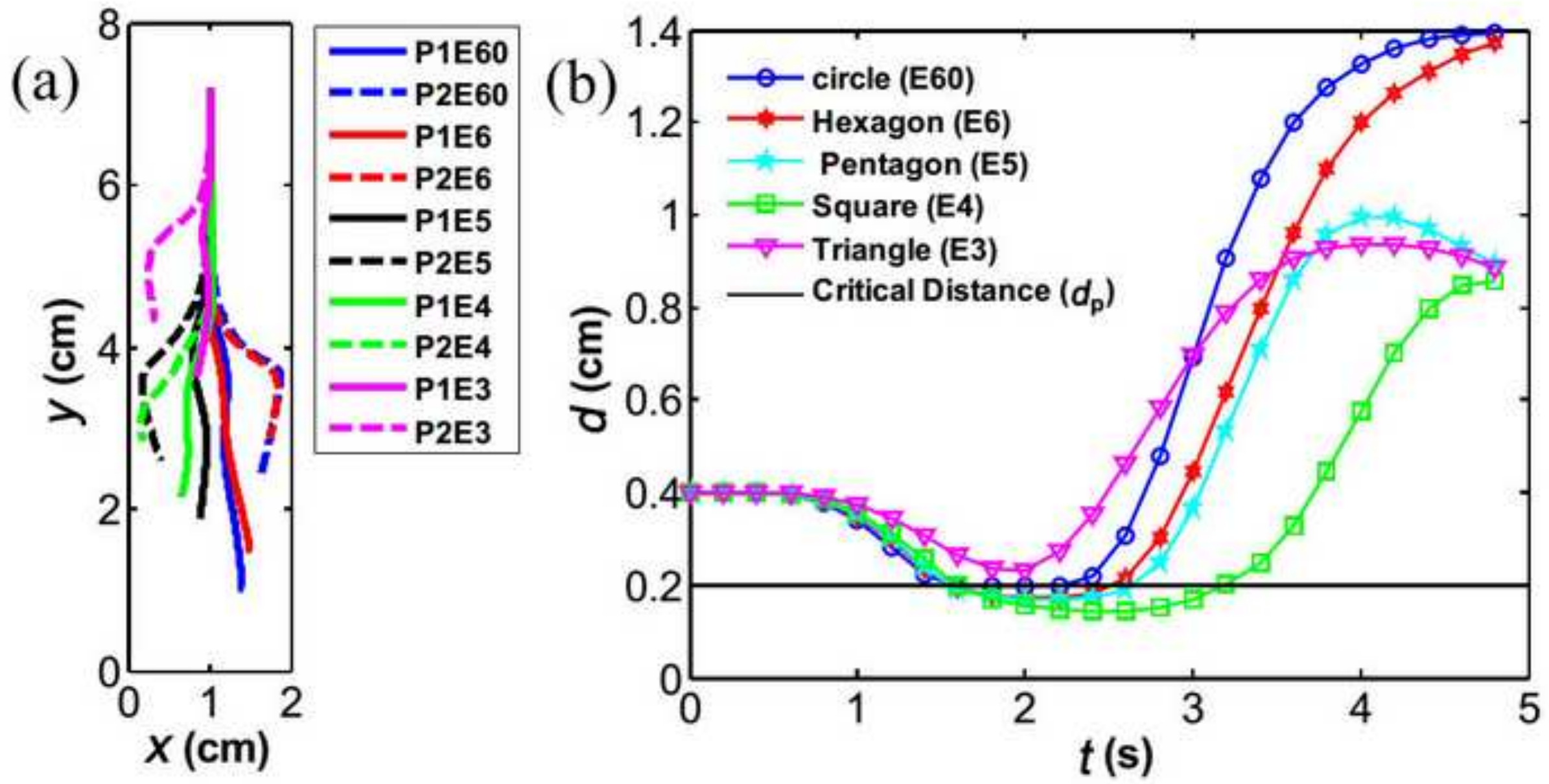

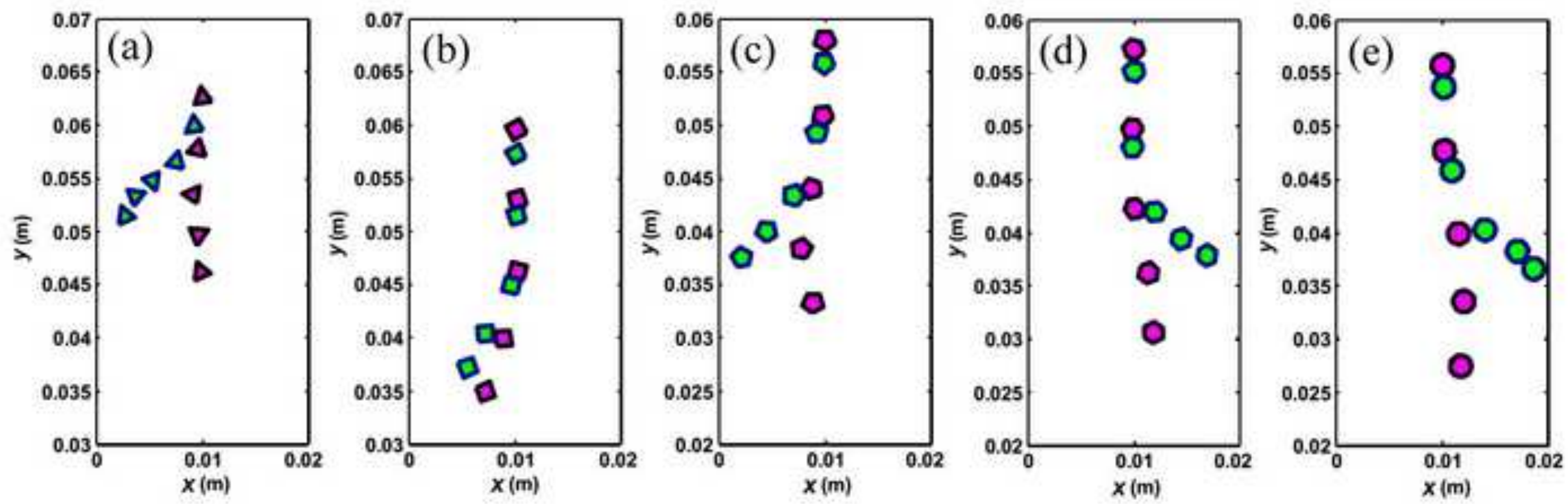

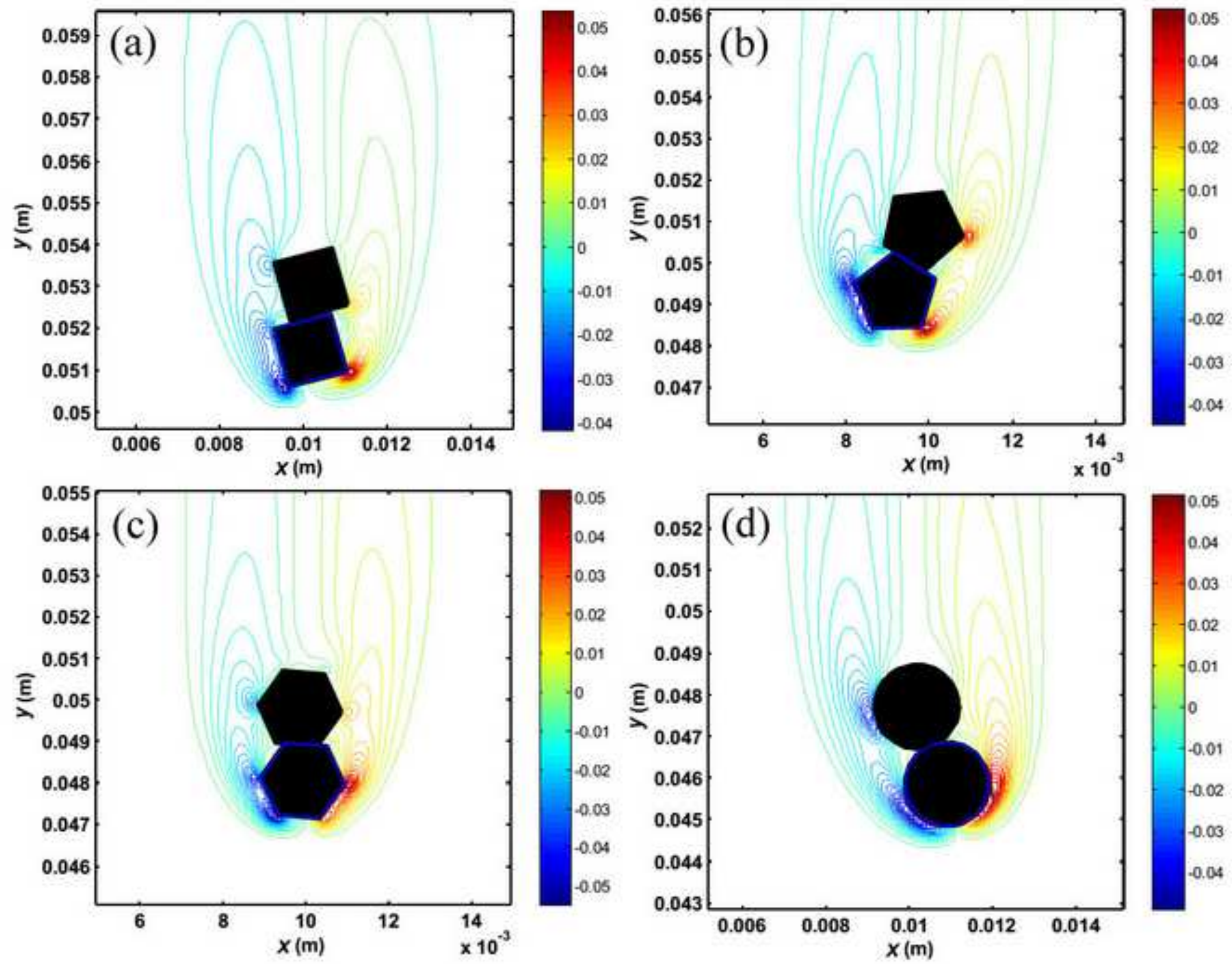
(a) Regular pentagons (b) Regular hesagons

(c) Circles
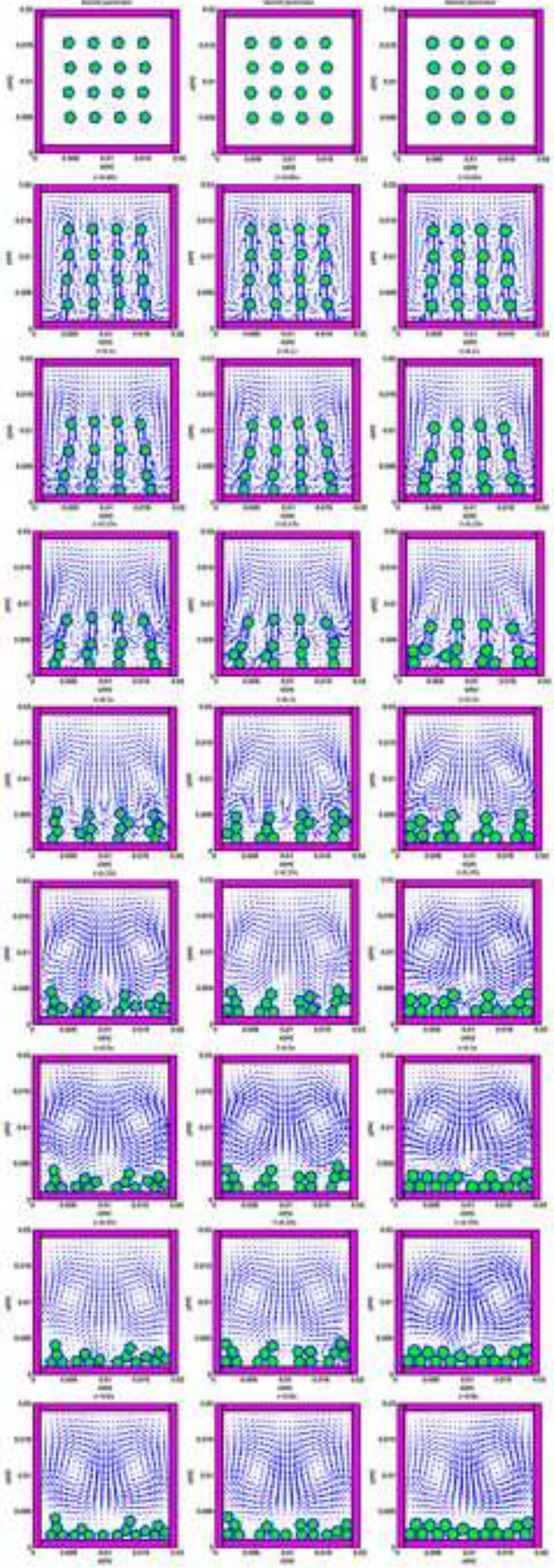

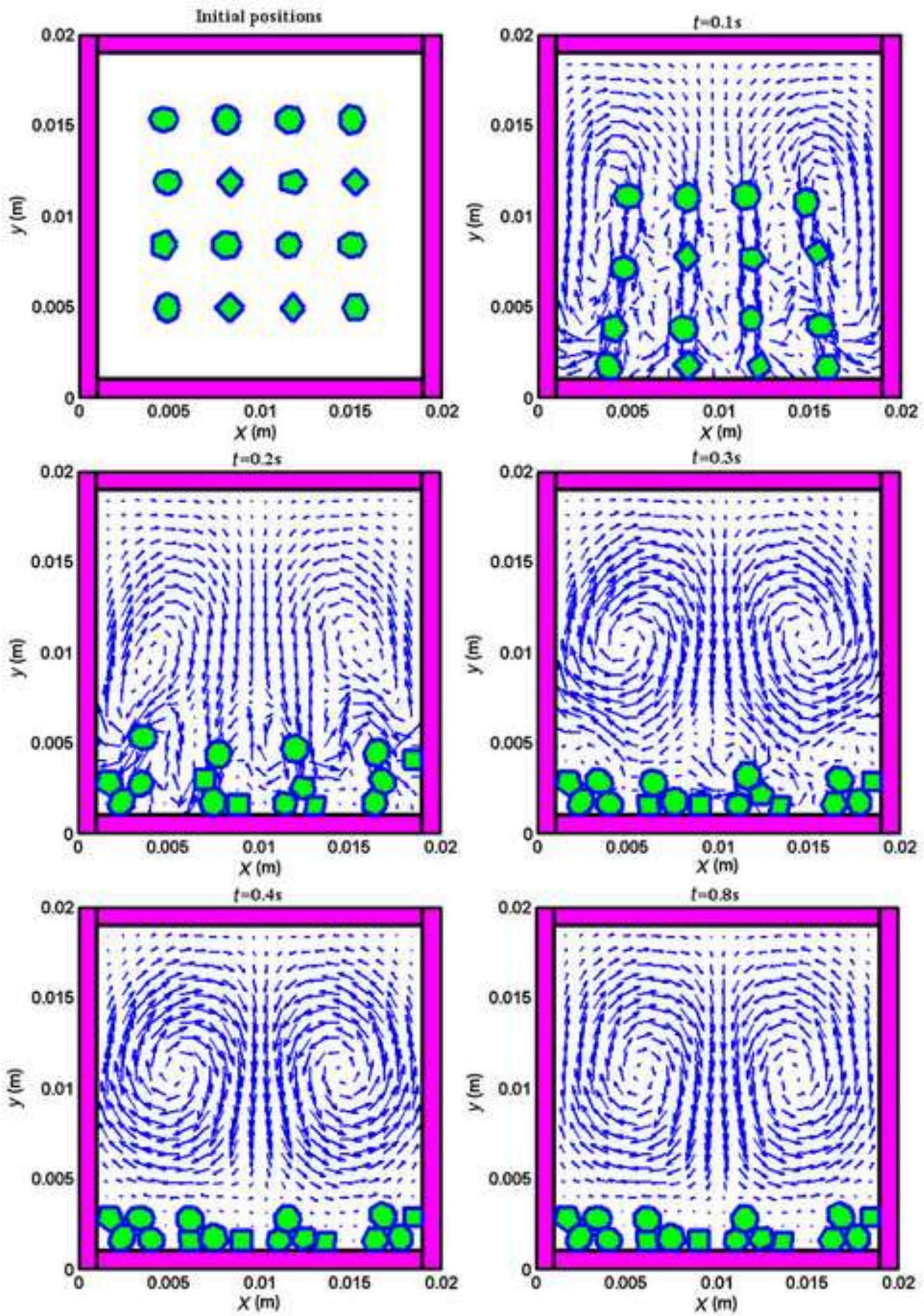


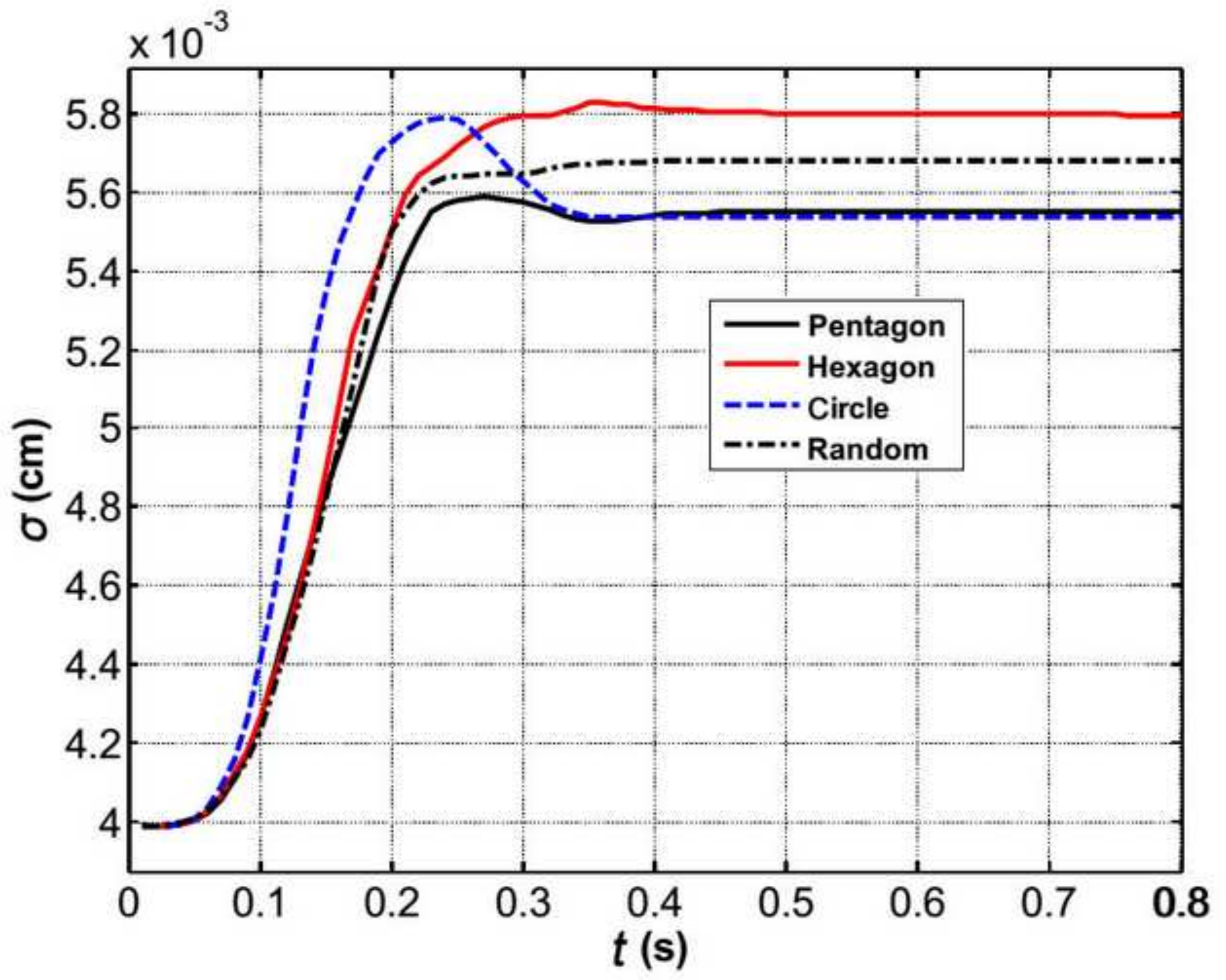



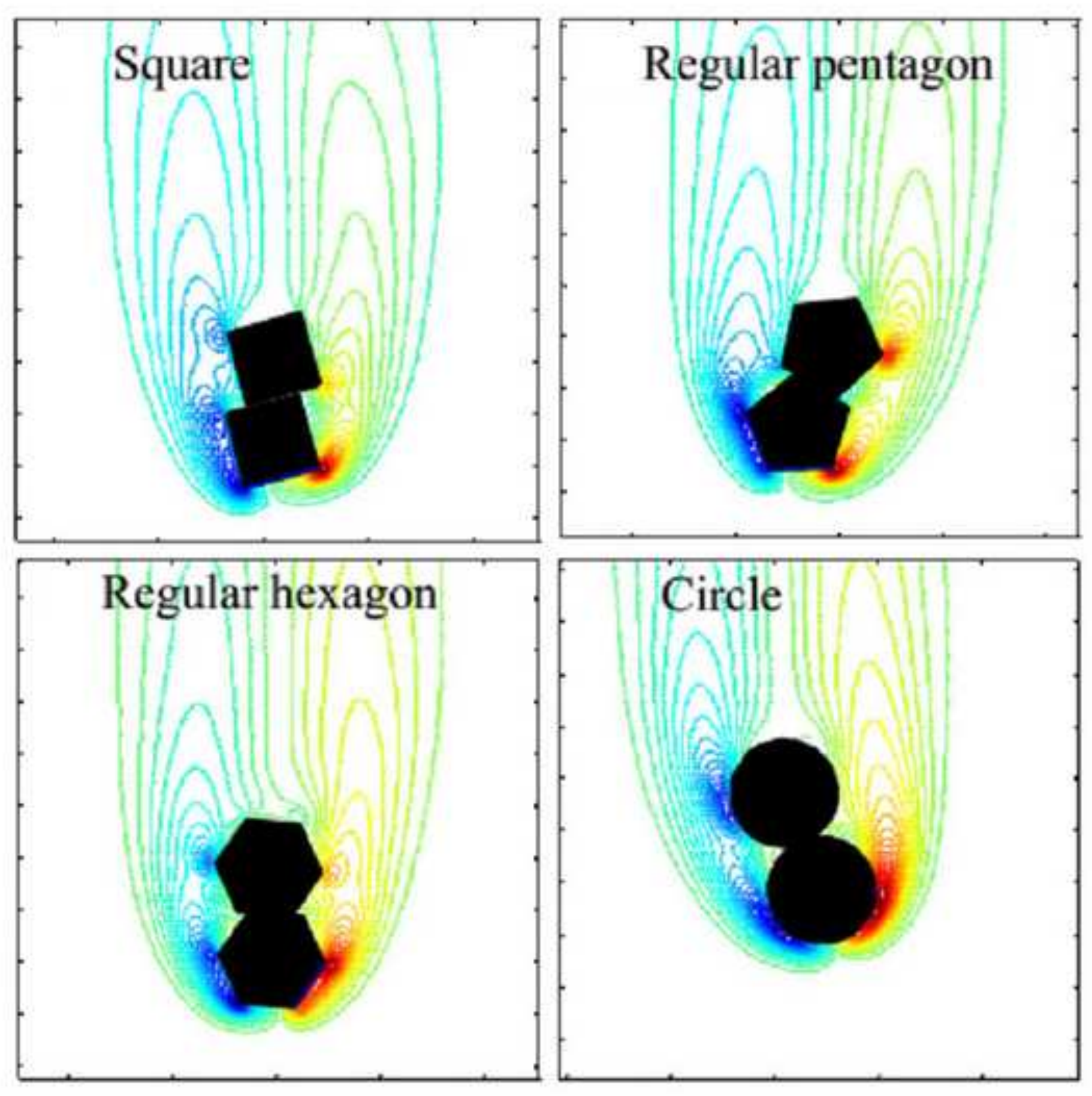